\title{
Thermodynamic perturbations in the X-ray halo of 33 clusters of galaxies observed with Chandra ACIS ${ }^{\star}$
}

\author{
F. Hofmann ${ }^{1}$, J. S. Sanders ${ }^{1}$, K. Nandra ${ }^{1}$, N. Clerc ${ }^{1}$, and M. Gaspari ${ }^{2,3, \star \star ~}$ \\ 1 Max-Planck-Institut für extraterrestrische Physik, Giessenbachstraße, 85748 Garching, Germany \\ e-mail: fhofmann@mpe.mpg.de \\ 2 Department of Astrophysical Sciences, Princeton University, Princeton, NJ 08544, USA \\ 3 Max-Planck-Institut für Astrophysik, Karl-Schwarzschildstraße, 85748 Garching, Germany
}

Received 9 July 2015 / Accepted 27 October 2015

\begin{abstract}
Context. In high-resolution X-ray observations of the hot plasma in clusters of galaxies, significant structures caused by AGN feedback, mergers, and turbulence can be detected. Many clusters have been observed by Chandra in great depth and at high resolution. Aims. With the use of archival data taken with the Chandra ACIS instrument, the aim was to study thermodynamic perturbations of the X-ray emitting plasma and to apply this to better understand the thermodynamic and dynamic state of the intracluster medium (ICM).

Methods. We analysed deep observations for a sample of 33 clusters with more than $100 \mathrm{ks}$ of Chandra exposure each at distances between redshift 0.025 and 0.45 . The combined exposure of the sample is $8 \mathrm{Ms}$. Fitting emission models to different regions of the extended X-ray emission, we searched for perturbations in density, temperature, pressure, and entropy of the hot plasma.

Results. For individual clusters, we mapped the thermodynamic properties of the ICM and measured their spread in circular concentric annuli. Comparing the spread of different gas quantities to high-resolution 3D hydrodynamic simulations, we constrained the average Mach number regime of the sample to $\mathrm{Mach}_{1 \mathrm{D}} \approx 0.16 \pm 0.07$. In addition we found a tight correlation between metallicity, temperature, and redshift with an average metallicity of $Z \approx 0.3 \pm 0.1 Z_{\odot}$.

Conclusions. This study provides detailed perturbation measurements for a large sample of clusters that can be used to study turbulence and make predictions for future X-ray observatories like eROSITA, Astro-H, and Athena.
\end{abstract}

Key words. galaxies: clusters: general - X-rays: galaxies: clusters - turbulence

\section{Introduction}

In the current picture of the evolution of the universe, clusters of galaxies have formed in the deep potential wells created by clumping of dark matter (DM) around remnant density fluctuations after the Big Bang. The majority of the mass in clusters is made of DM, which is only observed indirectly through its gravitational effects. The second component of clusters is baryonic matter consisting mainly of very thin hot plasma (the intracluster medium, ICM), heated by the gravitational accretion into the potential wells, and emitting strongest in X-ray wavelength because of its high temperatures. The smallest fraction of the mass is in the stellar content of the clustered galaxies, which is observable in visible light. The behaviour of DM in the cluster potential is believed to be well understood from cosmological simulations (e.g. Springel et al. 2005) and observations of gravitational lensing effects on visible light (Broadhurst et al. 1995; Kaiser et al. 1995; Allen et al. 2002; Bradač et al. 2006; Zhang et al. 2008; Mahdavi et al. 2013). The member galaxies of a cluster are well described as collisionless particles moving in the cluster potential by measuring the line-of-sight velocity dispersion

\footnotetext{
* Tables 2-67 are only available at the CDS via anonymous ftp to cdsarc.u-strasbg.fr (130.79.128.5) or via

http://cdsarc.u-strasbg.fr/viz-bin/qcat?J/A+A/585/A130

$\star \star$ Einstein and Spitzer Fellow
}

in the optical (see e.g. Zhang et al. 2011; Rines et al. 2013). The complex dynamic and thermodynamic processes in the hot ICM can be studied with X-ray observations. Other phases of the ICM have been studied at different wavelength with UV and $\mathrm{H} \alpha$ (e.g. McDonald et al. 2011) or radio observations (e.g. Dolag et al. 2001; Govoni et al. 2004).

Schuecker et al. (2004) first related fluctuations in the projected pressure maps of the hot, X-ray emitting, ICM of the Coma cluster to turbulence. Turbulence has been discussed as a significant heating mechanism (see Dennis \& Chandran 2005; Ruszkowski \& Oh 2010; Gaspari et al. 2012a), which is important to understand the heating and cooling balance (see cooling flow problem; Fabian 1994) in clusters and to estimate the amount of non-thermal pressure support (see e.g. simulations by Nelson et al. 2014). Zhuravleva et al. (2014) recently studied turbulence in the Perseus and Virgo cluster by analysing fluctuations in the surface brightness of the cluster emission. Asymmetries and fluctuations within thermodynamic properties of the hot plasma can be used to estimate the amount of turbulence (e.g. Gaspari \& Churazov 2013; Gaspari et al. 2014). This has been studied in the PKS 0745-191 galaxy cluster by Sanders et al. (2014). We applied a similar technique to the current sample of 33 clusters and compared our results to the findings of cluster simulations (e.g. Vazza et al. 2009; Lau et al. 2009).

The amount of turbulence in the hot ICM is hard to directly measure. Simulations of galaxy clusters predict turbulent 
motions of several hundreds of $\mathrm{km} \mathrm{s}^{-1}$ (e.g. Dolag et al. 2005; Nelson et al. 2014; Gaspari et al. 2014). Sanders et al. (2011), Sanders \& Fabian (2013), and Pinto et al. (2015) were able to obtain upper limits on the velocity broadening of spectra for a large sample of clusters. However current X-ray instruments only provide the spectral resolution needed to detect significant broadening due to turbulence in a few possible cases.

The basis for this study was the X-ray all-sky survey of the ROSAT mission (1990 to 1999; see Truemper 1982). The clusters identified in this survey (e.g. Böhringer et al. 2000, 2004) were followed up with the current generation X-ray telescopes Chandra, XMM-Newton, and Suzaku. For the substructure study we used observations of ROSAT clusters with the X-ray observatory on board the Chandra satellite, which delivers the best spatial and very good spectral resolution. Since its launch in 1999, Chandra has frequently been used to study clusters of galaxies as individual systems and as cosmological probes (e.g. Allen et al. 2004, 2008; Vikhlinin et al. 2009b,a). The telescope's high resolution showed an unexpected complexity of the ICM structure in many cases (e.g. Fabian et al. 2000; McNamara et al. 2000; Markevitch et al. 2000, 2002; Sanders et al. 2005; Fabian et al. 2006; Forman et al. 2007). We analysed a sample of clusters and mapped their thermodynamic properties based on the large archive of deep cluster observations. We specifically investigated perturbations in the thermodynamic parameters of the ICM, which according to recent high-resolution simulations can be used to trace turbulence in the ICM via the normalisation of the ICM power spectrum (e.g. in density; Gaspari et al. 2014). By measuring the slope of the power spectrum, we can constrain the main transport processes in the hot ICM, such as thermal conductivity (Gaspari \& Churazov 2013).

The paper is structured as follows: Sect. 2 describes the sample selection and data reduction; Sect. 3 describes the analysis of perturbations; in Sect. 4 the main results are presented; Sect. 5 contains results for individual systems; in Sect. 6 the findings are discussed; and Sect. 7 contains the conclusions.

For all our analysis we used a standard $\Lambda$ CDM cosmology with $H_{0}=71 \mathrm{~km} \mathrm{~s}^{-1} \mathrm{Mpc}^{-1}, \Omega_{\mathrm{M}}=0.27$ and $\Omega_{\Lambda}=0.73$ and relative solar abundances as given by Anders \& Grevesse (1989).

\section{Observations and data reduction}

We used archival observations with the Chandra Advanced CCD Imaging Spectrometer (ACIS; Garmire et al. 2003) using the imaging (-I) or spectral (-S) CCD array (about 0.1 to $10 \mathrm{keV}$ energy range). This instrument provides high spatial $\left(\sim 1^{\prime \prime}\right)$ and spectral resolution $(\sim 100 \mathrm{eV}$ full width half maximum, FWHM). The field of view (FOV) is limited, so that only the inner 5-10' of any cluster are homogeneously covered. See Sect. A for a list of all analysed clusters and their individual exposure times.

\subsection{Sample selection}

We based our sample selection on the NORAS (378 sources; see Böhringer et al. 2000), REFLEX (447 sources, see Böhringer et al. 2004), and CIZA (73 sources; see Ebeling et al. 2002) catalogues. They all have been derived from ROSAT observations, which deliver the only true imaging all-sky X-ray survey to date. The NORAS and REFLEX catalogues cover the regions north and south of the Galactic plane $\left( \pm 20^{\circ}\right)$, excluding the Magellanic Clouds. The CIZA sample covers the Galactic plane and thus adds some interesting clusters to our sample. However in the Galactic plane we have to deal with higher foreground absorption of X-rays (due to the column density $n_{\mathrm{H}}$ of the Galaxy).

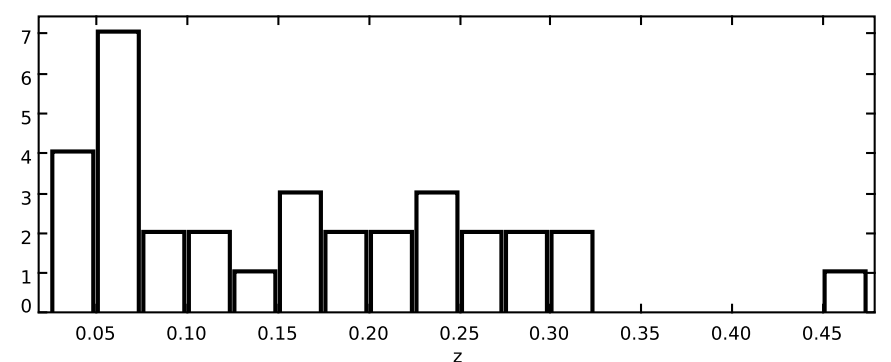

Fig. 1. Histogram of the redshift $(z)$ distribution of the final sample of 33 clusters.

Not all of these clusters have been observed with Chandra, but the predominantly X-ray bright clusters in which the structure of the ICM could be well studied have been observed with Chandra. We matched all Chandra ACIS observations available from the Chandra Data Archive (CDA ${ }^{1}$ on 2013-10-09) with cluster positions in the ROSAT catalogues mentioned above.

We set a luminosity cut on the $\sim 300$ clusters correlated with Chandra observations, and only added clusters to the sample that had a luminosity of more than $2.0 \times 10^{44} \mathrm{erg} / \mathrm{s}$ in the $0.1-2.4 \mathrm{keV}$ ROSAT energy band. We only accepted clusters and groups of galaxies with a redshift of $z \gtrsim 0.025$ to ensure all clusters fit reasonably well into the Chandra ACIS FOV. This excludes some nearby extended systems where larger radii are not homogeneously covered (e.g. the Coma cluster, see Vikhlinin et al. 2009b). After these selections we used all clusters with $\gtrsim 100$ ks raw Chandra exposure time. Our final sample consists of 33 X-ray bright, massive, nearby clusters of galaxies. The velocity dispersion of galaxies in the clusters is around $1000 \mathrm{~km} \mathrm{~s}^{-1}$ for most systems. The cluster halo masses within the overdensity radius $r_{500}$ range from $1 \times 10^{14} M_{\odot}$ to $2 \times 10^{15} M_{\odot}$ (Table 1 ). At $r_{500}$ the average density of the cluster is 500 times the critical density of the universe at the cluster redshift. The luminosity range is $(2-63) \times 10^{44} \mathrm{erg} / \mathrm{s}(0.1-2.4 \mathrm{keV} \mathrm{X}$-ray luminosity, see Tables A.1-A.3), the redshift ranges from 0.025 to 0.45 (see Fig. 1), and the total exposure analysed in this work is $\sim 8 \mathrm{Ms}$ (corresponding to more than 90 days of observations). For a list of observations used in this study, see Table B.1.

\subsection{Cluster maps}

For the Chandra data reduction we used a pipeline of various Python and $C++$ scripts to accurately map the structure of the ICM. The pipeline downloads all relevant datasets from the Chandra data archive (CDA) and reprocesses them using the Chandra standard data processing (SDP) with the Chandra Interactive Analysis of Observations software package (CIAO; Fruscione et al. 2006) version 4.5 and the Chandra Calibration Database (CalDB; Graessle et al. 2007) version 4.5.9.

A background light curve for each observation is created and times of high background are removed from the event files by iteratively removing times where the count rate is more than $3 \sigma$ from the median of the light curve. Via the CIAO tool acis_bkgrnd_lookup, we find a suitable blank-sky background file, which is provided by the Chandra X-ray centre (see e.g. Markevitch et al. 2003) and derive the background in each of the cluster observations. We correct residual spatial offsets between the individual observations, if necessary, by detecting point sources in each image with wavdetect and correlating the individual detection lists. Offsets in other event files

http://cxc.harvard.edu/cda/ 
Table 1. Sample properties.

\begin{tabular}{|c|c|c|c|c|c|c|c|}
\hline Cluster $^{a}$ & Abbrev. $^{a}$ & $\begin{array}{l}n_{\mathrm{H}} \\
{\left[10^{22} \mathrm{~cm}^{-2}\right]}\end{array}$ & $\begin{array}{l}\langle T \text { map }\rangle \\
{[\mathrm{keV}]}\end{array}$ & $\begin{array}{l}r_{\mathrm{FOV}}^{b} \\
{[\mathrm{Mpc}]}\end{array}$ & $\begin{array}{l}r_{500}{ }^{c} \\
{[\mathrm{Mpc}]}\end{array}$ & $\begin{array}{l}M_{500}{ }^{c} \\
{\left[10^{14} M_{\odot}\right]}\end{array}$ & $r_{\mathrm{FOV}} / r_{500}$ \\
\hline RX J1347-114 & rxj1347 & 0.046 & $14.7 \pm 0.125$ & 0.48 & $1.57 \pm 0.05$ & $17.7 \pm 1.8$ & 0.31 \\
\hline 1E 0657-56 & $1 \mathrm{e} 0657$ & 0.049 & $12.6 \pm 0.112$ & 0.98 & $1.58 \pm 0.06$ & $15.3 \pm 1.9$ & 0.62 \\
\hline A 2390 & a2390 & 0.062 & $10.8 \pm 0.104$ & 0.55 & $1.51 \pm 0.07$ & $12.4 \pm 1.8$ & 0.36 \\
\hline A 1689 & a1689 & 0.018 & $10.4 \pm 0.115$ & 0.41 & $1.52 \pm 0.07$ & $12.1 \pm 1.8$ & 0.27 \\
\hline A 401 & a401 & 0.099 & $8.6 \pm 0.071$ & 0.36 & $1.45 \pm 0.09$ & $9.5 \pm 1.7$ & 0.25 \\
\hline A 2204 & a2204 & 0.057 & $8.5 \pm 0.035$ & 0.42 & $1.39 \pm 0.08$ & $9.0 \pm 1.6$ & 0.30 \\
\hline A 2034 & a2034 & 0.015 & $8.3 \pm 0.107$ & 0.31 & $1.40 \pm 0.09$ & $8.8 \pm 1.6$ & 0.22 \\
\hline A 1413 & a1413 & 0.018 & $8.3 \pm 0.107$ & 0.36 & $1.38 \pm 0.08$ & $8.7 \pm 1.6$ & 0.26 \\
\hline A 2744 & a2744 & 0.014 & $8.7 \pm 0.229$ & 1.09 & $1.30 \pm 0.08$ & $8.6 \pm 1.5$ & 0.84 \\
\hline A 1835 & a1835 & 0.020 & $8.5 \pm 0.040$ & 0.42 & $1.32 \pm 0.08$ & $8.5 \pm 1.5$ & 0.32 \\
\hline PKS 0745-191 & pks0745 & 0.373 & $7.9 \pm 0.031$ & 0.40 & $1.37 \pm 0.09$ & $8.2 \pm 1.6$ & 0.29 \\
\hline А 665 & a665 & 0.043 & $7.3 \pm 0.137$ & 0.48 & $1.26 \pm 0.09$ & $6.9 \pm 1.5$ & 0.38 \\
\hline CYGNUS A & cygnusa & 0.272 & $6.9 \pm 0.022$ & 0.29 & $1.30 \pm 0.10$ & $6.8 \pm 1.5$ & 0.22 \\
\hline ZW 3146 & zw3146 & 0.025 & $7.0 \pm 0.064$ & 0.39 & $1.18 \pm 0.09$ & $6.3 \pm 1.4$ & 0.33 \\
\hline A 520 & a520 & 0.057 & $6.7 \pm 0.073$ & 0.79 & $1.20 \pm 0.09$ & $6.1 \pm 1.4$ & 0.66 \\
\hline A 1795 & a1795 & 0.012 & $6.2 \pm 0.008$ & 0.30 & $1.23 \pm 0.10$ & $5.8 \pm 1.4$ & 0.24 \\
\hline A 1650 & a1650 & 0.013 & $6.0 \pm 0.036$ & 0.28 & $1.20 \pm 0.10$ & $5.5 \pm 1.4$ & 0.23 \\
\hline A 3667 & a3667 & 0.044 & $5.8 \pm 0.025$ & 0.37 & $1.20 \pm 0.10$ & $5.3 \pm 1.4$ & 0.31 \\
\hline А 907 & a907 & 0.054 & $5.8 \pm 0.061$ & 0.28 & $1.14 \pm 0.10$ & $5.0 \pm 1.3$ & 0.24 \\
\hline A 521 & a521 & 0.049 & $5.9 \pm 0.177$ & 0.54 & $1.10 \pm 0.10$ & $4.8 \pm 1.3$ & 0.49 \\
\hline A 1995 & a1995 & 0.012 & $5.9 \pm 0.173$ & 0.31 & $1.06 \pm 0.09$ & $4.7 \pm 1.2$ & 0.29 \\
\hline A 2146 & a2146 & 0.030 & $5.7 \pm 0.031$ & 0.50 & $1.08 \pm 0.10$ & $4.6 \pm 1.2$ & 0.46 \\
\hline MS0735.6+7421 & $\mathrm{ms} 0735$ & 0.033 & $5.5 \pm 0.030$ & 0.30 & $1.08 \pm 0.10$ & $4.5 \pm 1.2$ & 0.28 \\
\hline MS $1455.0+2232$ & ms1455 & 0.032 & $5.1 \pm 0.055$ & 0.31 & $1.01 \pm 0.10$ & $3.9 \pm 1.2$ & 0.30 \\
\hline A 2199 & a2199 & 0.009 & $4.4 \pm 0.010$ & 0.19 & $1.05 \pm 0.12$ & $3.5 \pm 1.2$ & 0.18 \\
\hline A 496 & a496 & 0.038 & $4.3 \pm 0.012$ & 0.20 & $1.04 \pm 0.12$ & $3.4 \pm 1.2$ & 0.19 \\
\hline А 2597 & a2597 & 0.025 & $4.0 \pm 0.014$ & 0.21 & $0.98 \pm 0.12$ & $2.9 \pm 1.1$ & 0.21 \\
\hline 3C348 (HERCULES A) & $3 \mathrm{c} 348$ & 0.062 & $3.9 \pm 0.032$ & 0.23 & $0.94 \pm 0.12$ & $2.7 \pm 1.1$ & 0.25 \\
\hline A 1775 & a1775 & 0.010 & $3.7 \pm 0.047$ & 0.22 & $0.94 \pm 0.13$ & $2.6 \pm 1.1$ & 0.23 \\
\hline HYDRA A & hydraa & 0.047 & $3.5 \pm 0.009$ & 0.22 & $0.93 \pm 0.13$ & $2.4 \pm 1.1$ & 0.23 \\
\hline 2A $0335+096$ & 2a0335 & 0.175 & $2.9 \pm 0.005$ & 0.18 & $0.84 \pm 0.15$ & $1.8 \pm 1.0$ & 0.21 \\
\hline SERSIC 159-03 & sersic159 & 0.011 & $2.8 \pm 0.012$ & 0.17 & $0.82 \pm 0.15$ & $1.7 \pm 0.9$ & 0.20 \\
\hline A 2052 & a2052 & 0.027 & $2.3 \pm 0.002$ & 0.15 & $0.76 \pm 0.17$ & $1.3 \pm 0.9$ & 0.19 \\
\hline
\end{tabular}

Notes. ${ }^{(a)}$ Most commonly used cluster name and abbreviated catalogue names of clusters (compare to Tables A.1-A.3). Sorted on descending mass $\left(M_{500}\right){ }^{(b)}$ Maximum radius (from X-ray peak) covered in our analysis. ${ }^{(c)}$ Overdensity radii $r_{500}$ and $M_{500}$ were calculated using the masstemperature scaling relation from Vikhlinin et al. (2009a) for an estimate on the $r_{500}$ fraction covered in each object. We used $\langle T$ map $\rangle$ as input for the scaling relation and estimated errors assuming a $0.5 \mathrm{keV}$ systematic uncertainty (see scatter in Fig. 4).

are corrected by updating their aspect solution, using the deepest observation as a reference. With this procedure we ensure the best resolution of small-scale ICM variations. We create images from the event file of each dataset using an energy range of $0.5 \mathrm{keV}$ to $7.0 \mathrm{keV}$ and binning the image by a factor of two ( 1 pix $\sim 0.98^{\prime \prime}$ ). For each image, an exposure-map is created for an energy of $1.5 \mathrm{keV}$. After the analysis of the individual observations, all images, background, and exposure maps are merged. A second point source detection with wavdetect is run on the merged images and, after carefully screening the detection list, the point sources are masked from the image. In the following steps, the image is adaptively smoothed with snsmooth $=15$ and then binned into regions of equal signal-to-noise ratio $(\mathrm{S} / \mathrm{N})$ of 50 ( $>2500$ counts per bin) with the contour binning technique contbin (see Sanders 2006).

For the asymmetry (i.e. spread) analysis (Sect. 3.1), we generated maps with $S / N=25$ (>625 counts per bin) for clusters where we obtained less than 50 independent spatial-spectral bins from the $S / N=50$ analysis. For each of the bins a detector response is calculated and the count spectrum extracted. Spectra of the same detectors are added together (ACIS-I and ACIS-S separately) and fitted using C-Statistics in XSPEC version 6.11 (Cash 1979; Arnaud 1996) with the apec model for collisionally-ionised diffuse gas, which is based on the ATOMDB code v2.0.2 (Foster et al. 2012). The fit was done using a fixed foreground column density $\left(n_{\mathrm{H}}\left[\mathrm{cm}^{-2}\right]\right.$, see Table 1$)$, which is determined from the Leiden/Argentine/Bonn (LAB) survey of Galactic HI (based on Kalberla et al. 2005), and a fixed redshift from the ROSAT catalogues.

We only carried out the fit with $n_{\mathrm{H}}$ as a free parameter in the special case of PKS 0745-191 because of its location behind the Galactic plane and strong $n_{\mathrm{H}}$ variation within the FOV. The free parameters of the fit to the count spectrum are temperature $T$ [keV], the metal abundance $Z$ as a fraction of solar abundances (reference solar abundance $Z_{\odot}$ from Anders \& Grevesse 1989) and the normalisation $\eta\left[\mathrm{cm}^{-5} \operatorname{arcsec}^{-2}\right]$ of the fit, which is defined as

$\eta=10^{-14} /\left(4 \pi D_{\mathrm{A}}^{2}(1+z)^{2}\right) \int n_{\mathrm{e}} n_{\mathrm{p}} \mathrm{d} V$,

where $D_{\mathrm{A}}$ is the angular diameter distance to the source, $n_{\mathrm{e}}$ and $n_{\mathrm{p}}$ the electron and hydrogen densities integrated over the volume $V$. Assuming a spherical source, uniform density, and full ionisation with 10 per cent helium and 90 per cent hydrogen abundance (i.e. $n_{\mathrm{e}} \sim 1.2 n_{\mathrm{p}}$ ), the hydrogen density can be 

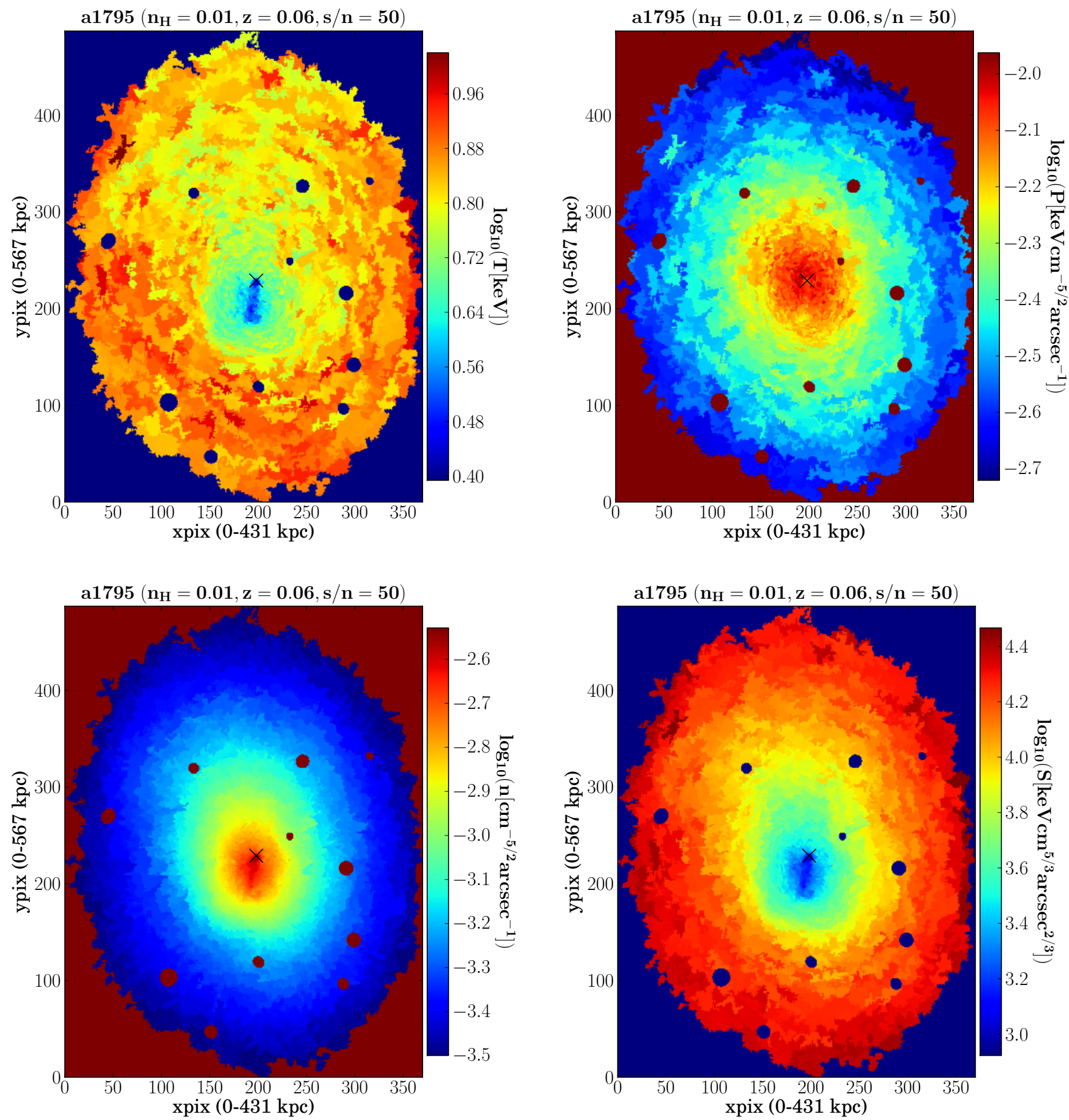

Fig. 2. From top left to bottom right: 2D maps of projected temperature, pressure, density, and entropy of A 1795. The cross denotes the X-ray peak and centre for profile analysis. Point sources and regions below the surface brightness cut are set to zero. xpix and ypix are pixels along RA and Dec direction and the overall range in kpc is given. Scale: 1 pix $\sim 1^{\prime \prime}$. The plot titles indicate the abbreviated cluster name, the average foreground column density $n_{\mathrm{H}}\left[\mathrm{cm}^{-2}\right]$, and the redshift.

calculated as

$n_{\mathrm{p}}=\sqrt{\frac{(1+z)^{2} 10^{14} \eta}{1.2 \Theta^{3} D_{\mathrm{A}}}} \equiv \xi \cdot \sqrt{\eta}$,

where $\Theta$ is the angular size of the source (see the ATOMDB webpage $^{2}$ for these equations). The factor $\xi$ changes for different

\footnotetext{
2 http://atomdb.org/faq.php
}

clusters and assumed geometries. We assumed $n_{\mathrm{p}} \sim \sqrt{\eta}$ within any given cluster. The fit parameters of each bin are translated into images to obtain maps of the temperature, metal abundance, and normalisation of the ICM.

From the 2D maps of the spectral fitting, we calculated a projected pseudo-density, pressure, and entropy in each spatial bin of the ICM emission. We assumed a constant line-of-sight depth for all spectral regions calculating pseudo-density $n$ as square 
root of the fit normalisation, normalised by region size,

$n \equiv \sqrt{\eta}\left[\mathrm{cm}^{-5 / 2} \operatorname{arcsec}^{-1}\right]$

pseudo-pressure as,

$P \equiv n \times$ temperature $\left[\mathrm{keV} \mathrm{cm}{ }^{-5 / 2} \operatorname{arcsec}^{-1}\right]$,

and pseudo-entropy as,

$S \equiv n^{-2 / 3} \times$ temperature $\left[\mathrm{keV} \mathrm{cm}{ }^{5 / 3} \operatorname{arcsec}^{2 / 3}\right]$.

We adopted a common definition of entropy for galaxy cluster studies, which is related to the standard definition of thermodynamic entropy s through

$s=k_{\mathrm{B}} \ln \left(S^{3 / 2}\left(\mu m_{\mathrm{p}}\right)^{5 / 2}\right)+s_{0}$,

with mean particle mass $\mu$ and proton mass $m_{\mathrm{p}}$ (see e.g. Voit 2005). Relative cooling times of the ICM are proportional to $n^{-1} \times$ temperature $^{1 / 2}\left[\mathrm{keV}^{1 / 2} \mathrm{~cm}^{5 / 2}\right.$ arcsec] where Bremsstrahlung emission dominates (see e.g. Sarazin 1986, and references therein).

All distances were calculated with the redshift given in the ROSAT cluster catalogues. All uncertainties are $1 \sigma$ confidence intervals unless stated otherwise. All further analysis only includes regions where the area-normalised normalisation of the fit was above $10^{-7} \mathrm{~cm}^{-5} \operatorname{arcsec}^{-2}$. This corresponds roughly to a surface brightness cut below in which there were insufficient counts for detailed spectral analysis. For an example refer to the maps of Abell 1795 in Fig. 2.

\section{Analysis of perturbations}

We were able to study the ICM in great detail for a large sample of clusters based on the very detailed spatial-spectral analysis of this sample of clusters with deep Chandra observations.

\subsection{Asymmetry measurement}

One of the main goals of this study was to better characterise the thermodynamic state of the ICM in individual clusters and to identify general trends in the whole sample. Important indicators of the state of the hot gas are fluctuations in thermodynamic properties. Gaspari \& Churazov (2013) and Gaspari et al. (2014), in recent high-resolution simulations, have shown a connection between these kinds of fluctuations and the Mach number of gas motion in the ICM.

We examined the asymmetry (i.e. the spread) of thermodynamic properties in concentric, circular annuli of radius $r$ around the peak X-ray emission. As input we used the S/N 50 ICM maps (see Sect. 2.2). For nine clusters (a907, ms1455, a521, a665, a2744, a1775, a1995, 3c348, zw3146), we used S/N 25 instead to obtain at least five radial spread bins with a minimum of five data points per bin for all clusters. Assuming the data points are scattered statistically as a result of their uncertainties $\sigma_{\text {stat }}$, we tested for intrinsic spread $\sigma_{\text {spread }}$ in the radial profiles (see e.g. Fig. 3).

To avoid contamination of the spread measurement by the slope, we modelled the radial profile by interpolating between several nodes. This modelled average profile can be described by a function $\mu\left(\mathrm{r}, \mu_{1}, \ldots, \mu_{\mathrm{N}}\right)$ with $N=7$. The nodes divide the cluster profile into bins with equal number of data points. We used a minimum of five data points per model node and

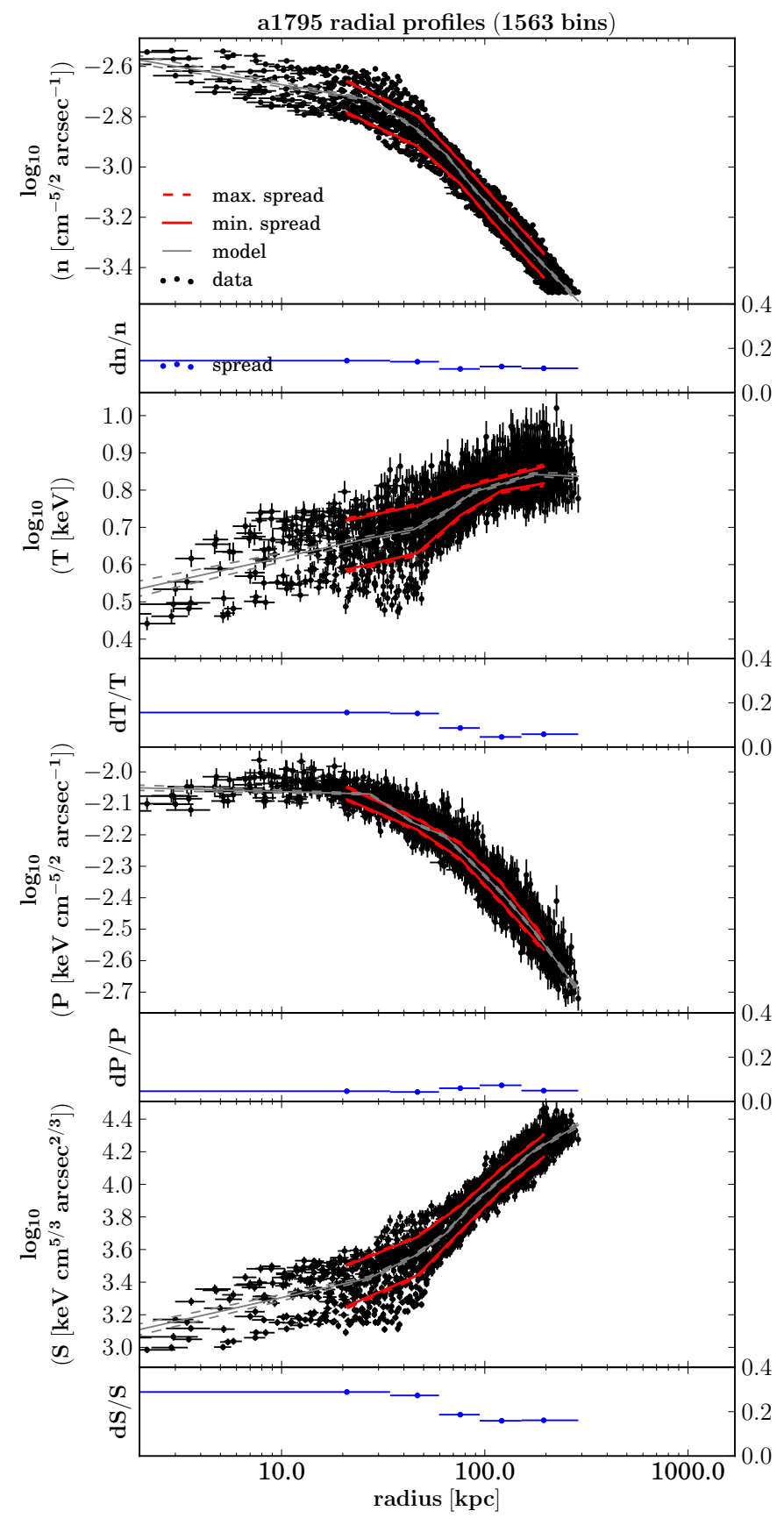

Fig. 3. Radial profiles of projected density, temperature, pressure, and entropy of A 1795. The centre is marked by a cross in Fig. 2. Error bars are the fit errors and the standard deviation of the radial distribution of the respective spatial-spectral bin. The plotted lines show limits on intrinsic scatter around an average seven-node model (grey lines) within the given radial range (see Sect. 3.1). The small panels show the measured fractional scatter $(M=5)$ with confidence and radial range.

if this criterion was not met we reduced the number of nodes. The intrinsic fractional spread is given by a function of the form $\sigma_{\text {spread }}\left(r, \sigma_{\text {spread, } 1}, \ldots, \sigma_{\text {spread,M }}\right)$. We performed three independent spread measurements, splitting the profiles into one, two, and five radial bins $(M=1,2,5)$ to measure the spread in the clusters with different radial resolutions.

For every data point, we obtained the mean value $\mu\left(r_{i}\right)$ of the thermodynamic profile at its radius $r_{\mathrm{i}}$ by interpolating between the model nodes (see grey lines in Fig. 3). The individual spread 
values of each data point $\sigma_{\text {spread }}\left(r_{\mathrm{i}}\right)$ are constant within a given radial bin (see spread profile in Fig. 3).

The intrinsic spread of cluster properties was estimated using a Markov chain Monte Carlo (MCMC) method implemented with emcee (see Foreman-Mackey et al. 2013) with 100 walkers, 1000 iterations, and a burn-in length of 1000 . The total scatter of data values (i) was assumed to follow a Gaussian distribution with standard deviation of $\sigma_{\text {tot }}$ calculated as

$\sigma_{\text {tot,i }}=\sqrt{\sigma_{\text {stat }, i}^{2}+\sigma_{\text {spread }}^{2}\left(r_{i}\right)}$.

For each iteration of the MCMC $\chi^{2}$ was calculated as

$\chi^{2}=\sum_{i=1}^{n} \frac{\left(D_{i}-\mu\left(r_{i}\right)\right)^{2}}{\sigma_{\mathrm{tot}, i}^{2}}$,

where $D_{i}$ are the individually measured values of the $\mathrm{n}$ data points (see Fig. 3). The mean model and spread values have $N+M$ free parameters. These parameters were varied via the MCMC method, giving the logarithm of the likelihood as the probability value for each iteration (see e.g. Hogg et al. 2010),

$\log L=-\frac{1}{2} \chi^{2}-\frac{1}{2} \sum_{i=1}^{n} \log \left(2 \pi \sigma_{\text {tot }, i}^{2}\right)$.

From this procedure we obtained a distribution of mean and spread values. We selected the best fit value for each parameter as the maximum of the distribution and estimated the uncertainty by giving the range containing 34 per cent of the obtained values on each side of the maximum. If the distribution was consistent with zero, we give the 68 per cent range as an upper limit.

The spread measurements performed with just one radial spread bin $(M=1)$ were used to compare the overall fractional spread $\mathrm{d} n, \mathrm{~d} T, \mathrm{~d} P$, and $\mathrm{d} S$ among clusters (see e.g. Figs. 6, 7, 10, and Table C.1) and constrain the general ICM properties. For additional comparisons of larger and smaller physical radii, we measured the spread inside and outside of $100 \mathrm{kpc}$ from the X-ray peak ( $M=2$, see e.g. $\mathrm{d} P_{\text {cen }}$ and $\mathrm{d} P_{\text {out }}$, Sect. 4.2). From the spread analysis with five radial spread bins $(M=5)$, we obtained profiles of the intrinsic fractional standard deviation of thermodynamic properties $\mathrm{d} n / n, \mathrm{~d} T / T, \mathrm{~d} P / P$, and $\mathrm{d} S / S$ (see Fig. F.1) in individual systems. The spread measurements are consistent with the results of a second Monte Carlo simulationbased technique, which was not based on Markov chains (similar to Sanders et al. 2014).

\subsection{Surface brightness substructures}

The emissivity of the ICM, and thus its surface brightness in $\mathrm{X}$-rays, is proportional to the plasma density squared (for a review, see e.g. Sarazin 1986). From the data reduction pipeline we obtained merged count and exposure images of the clusters. To remove any structure due to inhomogeneous exposure, we divided the count image by the exposure image and obtained an image of the count rate. To identify small surface brightness fluctuations, we enhanced the contrast of those images by unsharp-masking, a method commonly used in image analysis. This method is implemented by subtracting two versions of an image, smoothed by two different Gaussian filters, from each other. We subtracted an image smoothed with a Gaussian function of $\sigma=2$ pixel width from an image smoothed with a Gaussian function of $\sigma=5$ pixel width (see Fig. E.1). The obtained unsharp-masked images enhance surface brightness features, complementing the analysis of substructure in thermodynamic properties and highlighting disturbed systems.

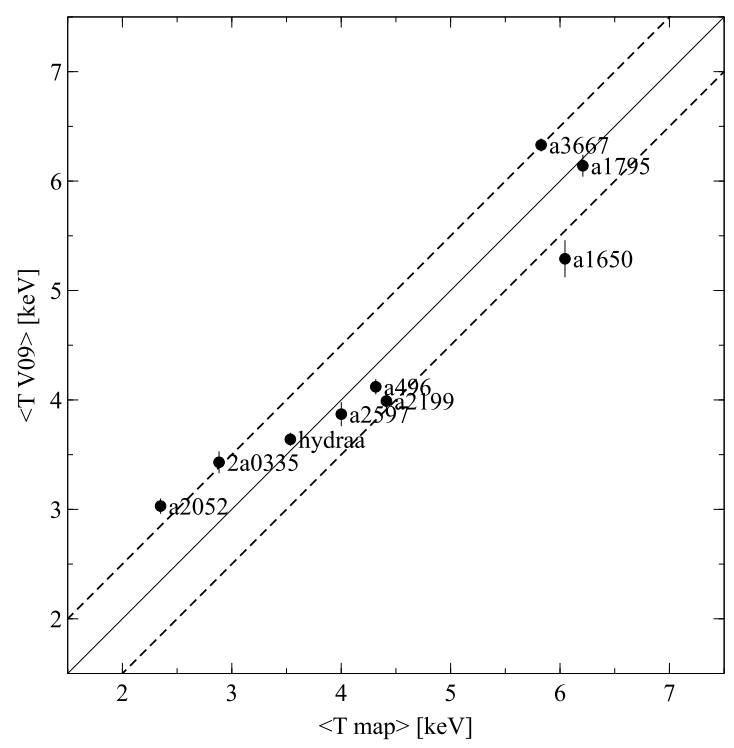

Fig. 4. Comparison of area- and error-weighted average 2D map temperatures $\langle T$ map $\rangle$ with overlapping low- $z$ sample temperatures of V09 (Vikhlinin et al. 2009a) showing a scatter of about $0.5 \mathrm{keV}$ around the one-to-one relation.

\subsection{Average cluster temperatures}

We calculated average cluster temperatures $\langle T$ map $\rangle$ as the areaand error-weighted mean value of all measurements. We computed the average of the bin temperature $(\langle T$ prof $\rangle$, see data points in Fig. 3), which is usually lower than $\langle T$ map $\rangle$ because $\langle T$ prof $\rangle$ is weighted more on emission than area. Hotter regions in the outskirts are generally larger with lower emission and the relatively colder, X-ray bright, central regions cover a smaller area.

We compared our approach for estimating overall cluster temperatures with previous studies like V09 using (using Chandra; Vikhlinin et al. 2009a) or HiFLUGS (using ROSAT; Reiprich \& Böhringer 2002), as shown in Figs. 4 and 5. Figure 4 shows significant scatter of up to $0.5 \mathrm{keV}$ between V09 and this study. This is expected since we use a different approach by averaging many independently fitted bins weighting by area and error bars rather than by counts. The averaging of many different bins results in small error bars on our average temperature. Figure 5 shows similar scatter when accounting for the larger error bars on the ROSAT measurements. We also use different extraction radii than our comparison studies, which influences the measured temperature.

To estimate the mass range of the cluster sample (see Table 1), we used the mass-temperature scaling relation from Vikhlinin et al. (2009a) with the $\langle T$ map $\rangle$ temperatures as input to calculate the overdensity radius $r_{500}$ and the total mass $M_{500}$ included by this radius. We accounted for uncertainties in the scaling by assuming a systematic temperature uncertainty of $0.5 \mathrm{keV}$ (see scatter in Fig. 4). The average map temperature (area weighted) is comparable to the core-excised average temperature used for the scaling by Vikhlinin et al. (2009a).

\subsection{Table description}

The main results of the analysis of this study are summarised in separate tables for each cluster. We created 2D maps from the merged observations of every cluster and measured the 


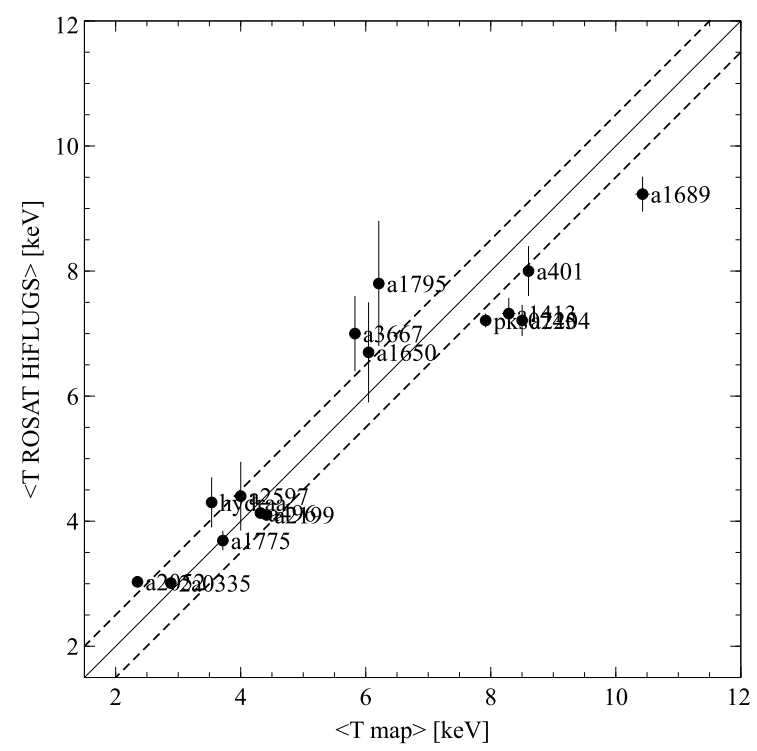

Fig. 5. Comparison of area- and error-weighted average 2D map temperatures $\langle T$ map $\rangle$ with HiFLUGS temperatures (Reiprich \& Böhringer 2002) from ROSAT observations for the clusters overlapping with our sample. The dashed lines show a one-to-one relation with a scatter of $0.5 \mathrm{keV}$.

asymmetries in thermodynamic parameters in circular concentric annuli around the centre.

\subsubsection{Map tables}

The primary data products of this analysis are $2 \mathrm{D}$ maps of the thermodynamic properties of the ICM based on the merged observations of ACIS-S and ACIS-I for every cluster in the sample (see Sect. 2.2).

Tables 2-34 contain the 2D map information (one table per cluster). Each table lists the properties for every pixel in the cluster maps $\left(1\right.$ pix $\left.\sim 0.98^{\prime \prime}\right)$. The map tables contain the following: $\mathrm{X}$ (east-west direction) and $\mathrm{Y}$ (south-north direction) position $(x, y$, Cols. 1,2$)$ of the pixel; index of the independentlyfitted, spatial-spectral bin it belongs to (binnum, Col. 3); photon counts (cts, Col. 4); the background counts (bgcts, Col. 5); effective exposure time (exp, Col. 6); temperature and its upper and lower limits ( $T$, Tup, Tlo, Cols. 7-9); relative metallicity and limits (Z, Zup, Zlo, Cols. 10-12); fit normalisation and limits (norm, normup, normlo, Cols. 13-15); redshift (redshift, Col. 16); foreground column density $n_{\mathrm{H}}(\mathrm{NH}, \mathrm{Col} 17)$; distance from the centre in pixels, arc seconds, and kpc (cen_dist, rad_arcsec, rad_kpc, Cols. 18-20); angle with respect to the west direction (cen_angl, Col. 21); calculated projected pressure and symmetric uncertainty $\left(P, P \_\right.$err, Cols. 22,23$)$; projected entropy and uncertainty (S, S_err, Cols. 24, 25); and density and uncertainty $\left(n, n \_\right.$err, Cols. 26, 27). All uncertainties are on $1 \sigma$ confidence level.

\subsubsection{Asymmetry tables}

The secondary data products are based on the 2D maps and contain the measured spread (i.e. asymmetry, deviation from radial symmetry of the thermodynamic parameters; see $M=5$ in Sect. 3.1).

Tables 35-67 provide the measured properties in the concentric annuli for one cluster each. They contain the average

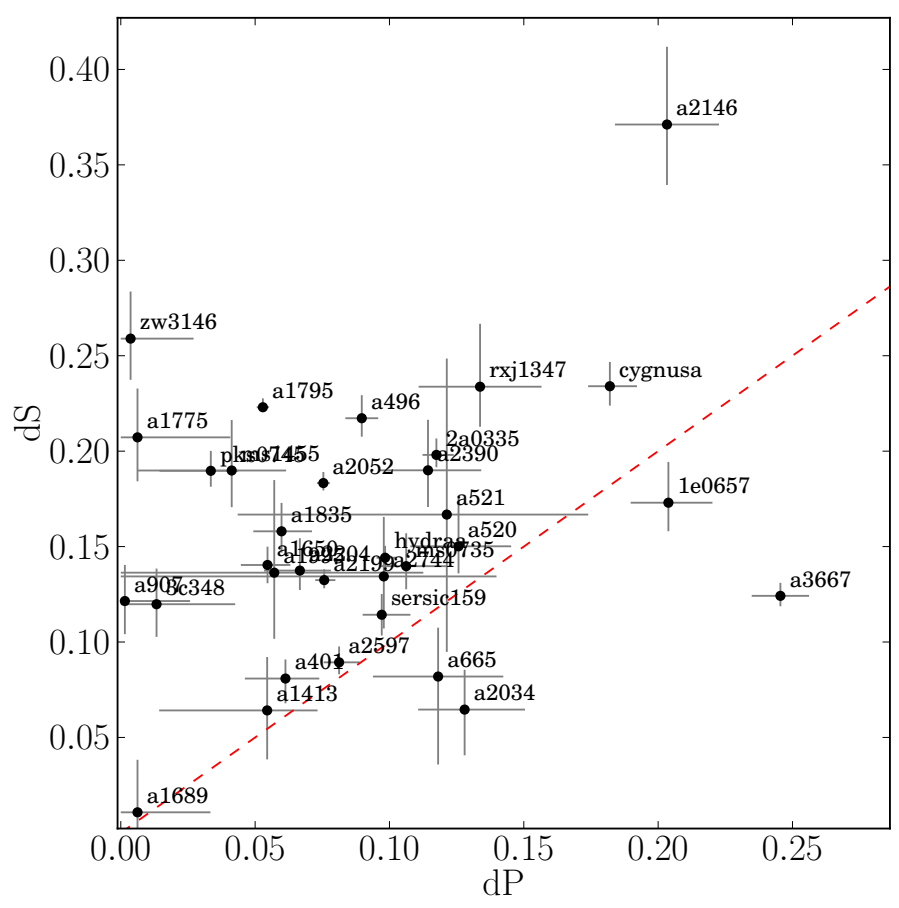

Fig. 6. Comparison of average projected pressure and projected entropy fluctuations for all clusters in the sample. The dashed line represents a one-to-one relation. The plot suggests that entropy fluctuations dominate in most clusters of the sample. Error bars are the statistical uncertainty from the MCMC measurements.

radius of the annulus (rr, Col. 1), average bin-temperature $\langle T\rangle$ and uncertainty (T, Te, Cols. 2, 3), and average bin metallicity and uncertainty (Z, Ze, Cols. 4, 5). For the intrinsic fractional spread values in projected properties, best-fit value and $1 \sigma$ upper and lower confidence limits are provided. Spread measurements contain the intrinsic spread in pressure with upper and lower limits ( $\mathrm{d} P, \mathrm{~d} P$ _eu, $\mathrm{d} P$ _el, Cols. 6-8), entropy with limits (d $S, \mathrm{~d} S \_$eu, $\mathrm{d} S \_$_el, Cols. 9-11), density with limits ( $\mathrm{d} n, \mathrm{~d} n \_$eu, $\mathrm{d} n \_$el, Cols. 12-14), and temperature with limits (dT, d $T \_$eu, dT_el, Cols. 15-17).

\section{Results}

The detailed analysis of this sample of clusters enabled us to derive information about the thermodynamic state of the ICM in individual clusters and the whole sample in general.

\subsection{Perturbations in thermodynamic properties}

We studied the average fluctuations in thermodynamic properties of all clusters in the sample using the $M=1$ spread calculations from Sect. 3.1. Comparing the average measurements for all systems in the sample enabled us to find general trends.

Figure 6 indicates that, on average, entropy fluctuations (fractional spread, $\mathrm{d} S$ ) dominate pressure fluctuations (fractional spread, $\mathrm{d} P$ ). For some clusters we only obtained upper limits (e.g. A 1689). There are some outliers (e.g. A 2146 and A 3667), which are heavily disturbed systems (see Sect. 6.1). Overall most clusters lie off a one-to-one correlation with an average of 16 per cent fluctuations in entropy and 9 per cent in pressure (see Table 68).

Figure 7 shows a smaller offset between the average density fluctuations (fractional spread, $\mathrm{d} n$ ) and temperature fluctuations 


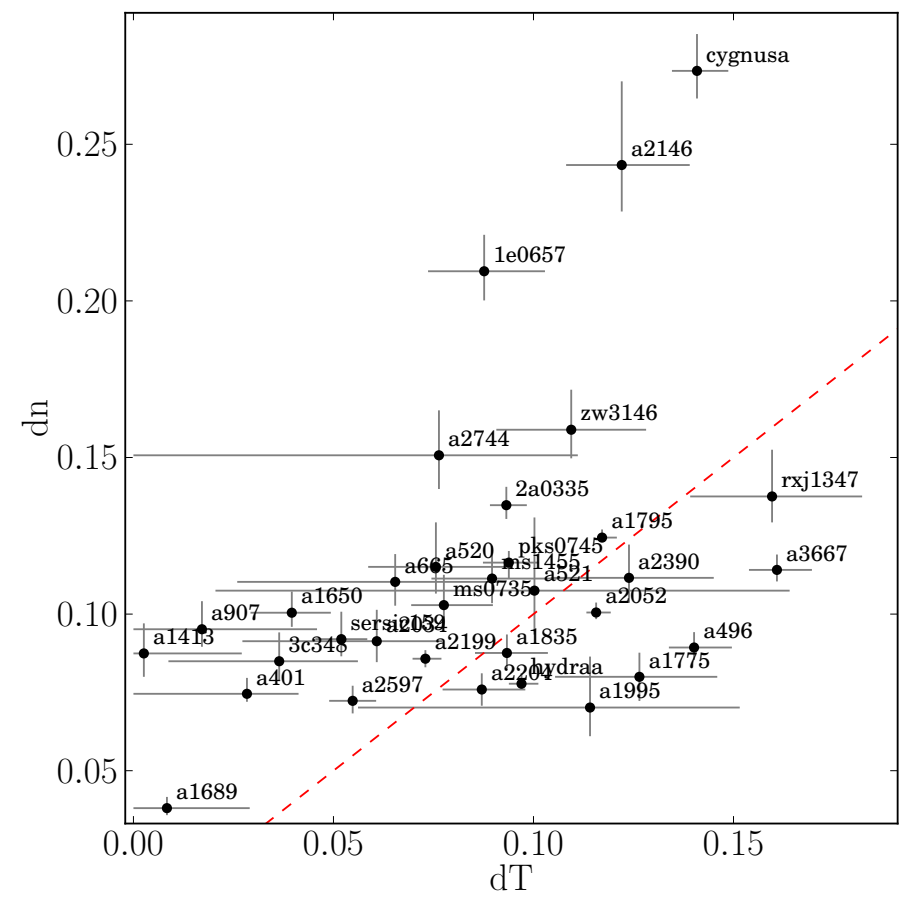

Fig. 7. Comparison of average temperature and projected density fluctuations for all clusters in the sample. The dashed line represents a one-to-one relation. Error bars are the statistical uncertainty from the MCMC measurements.

Table 68. Average perturbations.

\begin{tabular}{llll}
\hline \hline$\langle\mathrm{d} P\rangle^{a}$ & $\langle\mathrm{~d} S\rangle^{a}$ & $\langle\mathrm{~d} n\rangle^{a}$ & $\langle\mathrm{~d} T\rangle^{a}$ \\
\hline $0.09 \pm 0.06$ & $0.16 \pm 0.07$ & $0.11 \pm 0.05$ & $0.09 \pm 0.04$ \\
\hline
\end{tabular}

Notes. ${ }^{(a)}$ Average fractional perturbations in thermodynamic properties of the 33 sample clusters. Standard deviation as confidence range.

(fractional spread, $\mathrm{d} T$ ). We measure a significant spread in $\mathrm{d} n$ for every cluster in the sample, but only obtain upper limits on the $\mathrm{d} T$ spread for some. There are some outliers that show very strong density fluctuations (e.g. A 2146, 1E 0657-56, and Cygnus A). Overall, most clusters are close to a one-to-one correlation with an average of 11 per cent fluctuations in density and 9 per cent in temperature (see Table 68).

\subsection{Perturbations on different scales}

We found evidence for larger pressure perturbations $\mathrm{d} P$ at larger scales. Figure 8 shows a clear separation of the distribution for central regions $\lesssim 100 \mathrm{kpc}$ (cen) from the centre and regions beyond (out). To compare the distribution of spread values on different scales, we used the asymmetry measurements from Sect. 3.1, where the cluster profile is divided at $\sim 100 \mathrm{kpc}$. The division at $100 \mathrm{kpc}$ was chosen, after visual inspection, as a robust separation radius between central substructure and outer more homogeneous regions. We did not choose the regions relative to $r_{500}$ to ensure we are testing the same physical scale of the ICM fluctuations.

We used a bootstrapping re-sampling technique, calculating the mean value for 1000 permutations with repetition, to estimate the mean of the distributions. The contours are at 15 , 50 , and 85 percent of the maximum of the obtained distribution of mean values. In addition, Fig. 8 contains the output
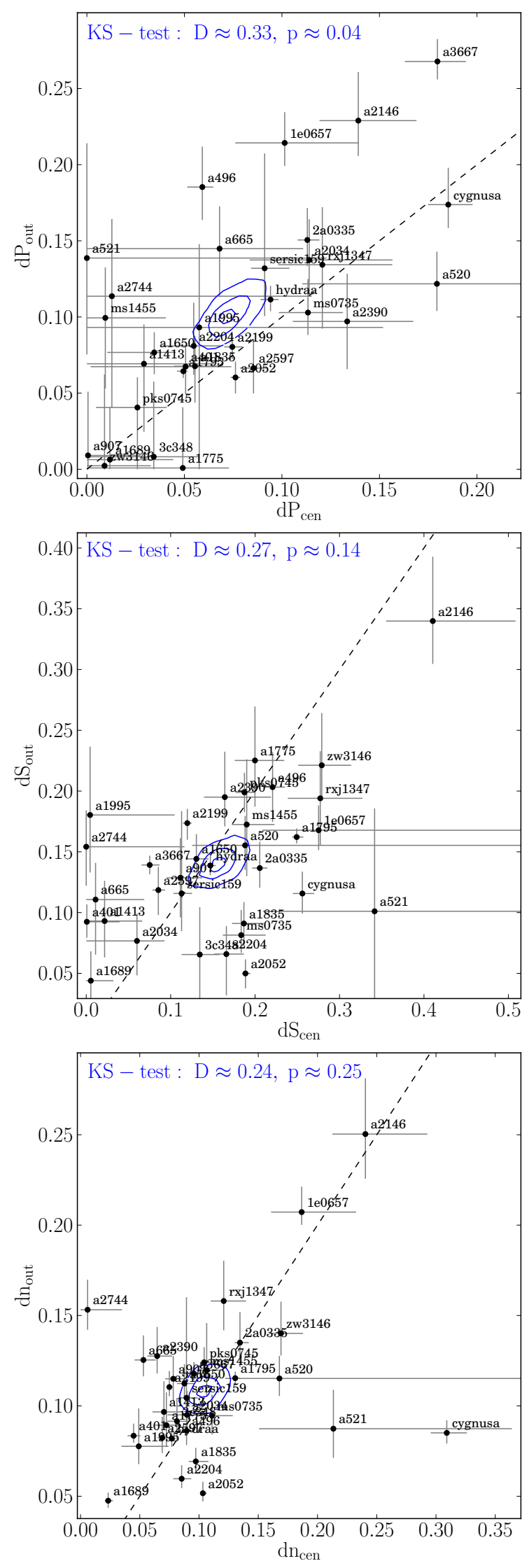

Fig. 8. Comparison of pressure (top), entropy (middle), and density perturbations (bottom) in the central $\lesssim 100 \mathrm{kpc}$ and outer $\gtrsim 100 \mathrm{kpc}$ regions. Confidence regions of the mean value as contours $(85,50$, and 15 per cent of peak value). KS test results on top. One-to-one correlation as dashed line. 
F. Hofmann et al.: Thermodynamic perturbations in galaxy clusters

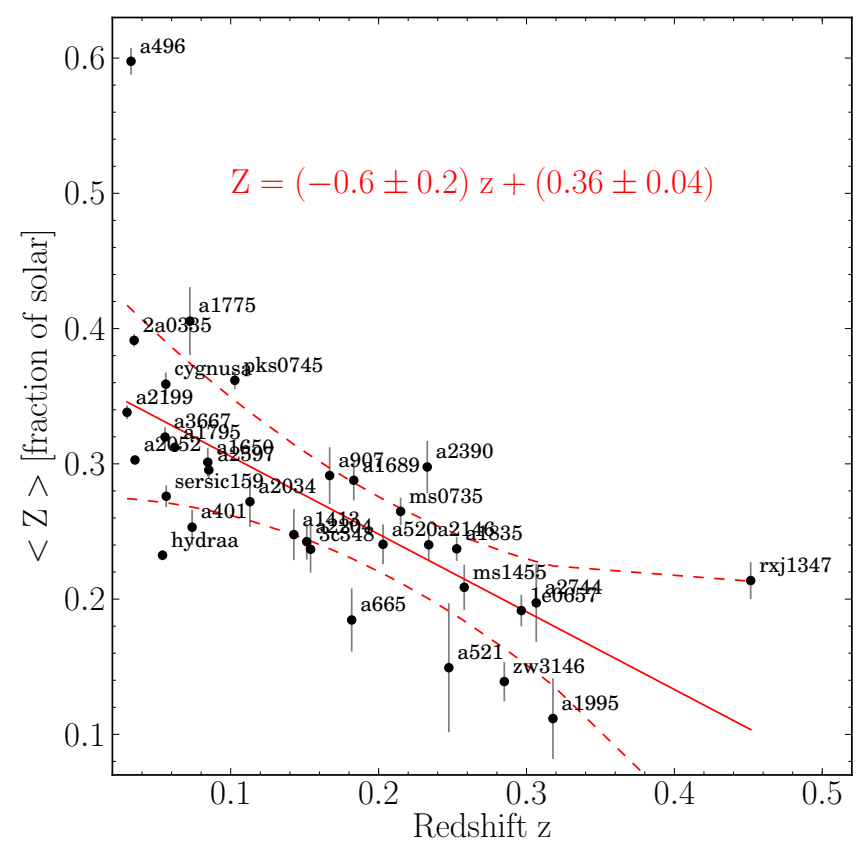

Fig. 9. Comparison of the cluster redshift $z$ and the area- and errorweighted average 2D map metallicity measured in the ICM (full radial range). The red line and equation show the best-fit linear correlation. Dashed lines indicate the $1 \sigma$ scatter around the best fit. Error bars are the statistical uncertainty of the weighted average.

of a Kolmogorov-Smirnov (KS) test to quantify the difference between the cen and out distributions. The $\mathrm{D}$ value states the maximum fractional offset between the cumulative distribution graphs and the $\mathrm{p}$ value is the probability of the null hypothesis. This means the probability that the $\mathrm{d} P$ distributions are different is 96 per cent and thus just above the $2 \sigma$ level. The offset in $\mathrm{d} P$ is dominated by low $\mathrm{d} P$ data points and decreases to about $1 \sigma$ level when only including data points above $0.05 \mathrm{~d} P$. For the thermodynamic properties $\mathrm{d} S, \mathrm{~d} n$, and $\mathrm{d} T$, there is no significant difference between inner and outer radii.

\subsection{Metallicity correlations}

We found an anti-correlation between the average temperatures $(\langle T$ map $\rangle$, see Sect. 3.3) of the clusters and their average metallicity. The best-fit linear correlation is $Z / Z_{\odot}=-(1.0 \pm$ $0.7) T / 100 \mathrm{keV}+(0.34 \pm 0.06)$. The average metallicity of the clusters have been weighted by area and error in the same way as the average map temperatures in Sect. 3.3. A similar correlation can be found within the individual clusters (see maps in Sect. 3.4.1). By repeating the same slope analysis for the inner $(\lesssim 100 \mathrm{kpc})$ and outer $(\gtrsim 100 \mathrm{kpc})$ regions of the cluster, we found that the average metallicity is higher in the central regions and the slope of the $T-Z$ anti-correlation is steeper. Testing different weighting methods for the average temperature and metallicity, we obtained consistent correlations with some scatter. Temperature is also correlated to the redshift of the clusters in this sample (more luminous and massive systems at higher redshift due to selection bias).

We investigated the redshift-metallicity $(z-Z)$ relation and found the correlation with redshift to be tighter than with temperature (see Fig. 9). The best-fit linear correlation of metallicity and redshift is $Z / Z_{\odot}=-(0.6 \pm 0.2) z+(0.36 \pm 0.04)$ (see Fig. 9). There was no evidence that the $z-Z$ anti-correlation is steeper in the central regions. The average metallicity of the sample is $Z \approx 0.3 \pm 0.1 Z_{\odot}$. The significance of a correlation was estimated using a re-sampling technique, performing a linear fit on random subsamples of 17 clusters and using the mean value and $1 \sigma$ width as best fit and error range of slope and normalisation.

\section{Individual clusters}

In addition to the sample properties, the data contain important information about the properties of individual systems. The sample consists of clusters with a wide range of different structures from more relaxed systems, such as A 496 and A 2199, to disturbed clusters, such as 1E 0657-56 and A 2146. We highlight some special cases below. Temperature maps, unsharp-masked images, and radial profiles of thermodynamic properties can be found in Sect. D, E, and F.

Abell 1795 has the deepest exposure in our sample and thus the most detailed spatial-spectral information on the ICM could be derived in this system. Oegerle \& Hill (1994) found that its central $\mathrm{cD}$ galaxy has a peculiar radial velocity of $150 \mathrm{~km} \mathrm{~s}^{-1}$ within the cluster, while they measured the velocity dispersion of cluster members at $\sigma=920 \mathrm{~km} \mathrm{~s}^{-1}$. According to Fabian et al. (2001), the simplest explanation for the visible soft $\mathrm{X}$-ray filament would be a cooling wake behind the $\mathrm{cD}$ galaxy (approximate position at the cross in Fig. 2, filament extending to the south), which is oscillating in the DM potential of the galaxy cluster. The centre is surrounded by linear surfacebrightness features that might be remnants of past mergers with subhalos or created by the outburst of a strong active galactic nucleus (AGN; see e.g. Markevitch et al. 2001; Ettori et al. 2002; Walker et al. 2014; Ehlert et al. 2015).

The deep unsharp-masked count images in this study show the central surface-brightness features at higher significance than previous studies (see Fig. E.1). The maps (Fig. 2) contain 1563 bins with a $\mathrm{S} / \mathrm{N}$ count ratio of 50 . The detailed radial profiles of the projected thermodynamic properties (Fig. 3) were the basis for the radial asymmetry measurements. Entropy perturbations seem to dominate throughout the cluster, suggesting that the 1D Mach number is comparable to the variance of entropy (see below, Sect. 6.2). The perturbation measurements are influenced by the presence of a cooler X-ray filament (inner $\sim 40 \mathrm{kpc}$ ) and projection-effects (see Sect. 6.1). The central filament seems to increase the spread in density, temperature, and entropy, but pressure seems unaffected (see scatter in Fig. 3). The profiles follow the average trend of higher pressure perturbations in the outskirts (see Sect. 4.2). The findings confirm and improve the detection of many surface-brightness features at the centre of the cluster. The detailed analysis of thermodynamic perturbations support a model where isobaric processes dominate the central ICM.

1E0657-56 (the Bullet Cluster) offers an almost edge-on view of two massive merging subclusters (Markevitch et al. 2002). It was possible to find a significant offset between the total mass profile from weak-lensing and the X-ray emission of the hot ICM and thus make a very convincing case for the existence of DM (Markevitch et al. 2004; Clowe et al. 2004, 2006; Randall et al. 2008).

We found the strongest surface-brightness fluctuations around the prominent Mach cone from the impact of the smaller subcluster (see Fig. E.1). The density fluctuations in the cluster are among the highest in the observed sample (see Fig. 7). Pressure fluctuations around the Bullet are significantly weaker 
than at larger radii (see Fig. F.1). Major merger shocks and AGN feedback are not modelled in the Gaspari et al. (2014) simulations and, thus, in those cases, the direct connection between Mach number and fluctuations in thermodynamic parameters might change (see Sect. 6.1). 1E0657-56 has the second highest $\langle T$ map $\rangle$ in the sample (after RXJ1347-114) and the lowest average metallicity (see Fig. 9). The Bullet Cluster has a particularly flat pressure profile with large scatter when compared to other clusters. In the most disturbed systems, the pressure profile is flat and does not drop with radius.

Abell 2052 hosts an extended region of colder ICM at its centre caused by rising colder gas due to strong AGN feedback from the central $\mathrm{cD}$ galaxy. The radio source connected to the central AGN and its effect on the surrounding ICM have been studied in detail by Blanton et al. (2001, 2003, 2011). Feedback from the radio source pushes the X-ray emitting gas away from the centre and creates a sphere of enhanced pressure around the central region. Machado \& Lima Neto (2015) investigated different merger scenarios in recent simulations of the cluster.

In our measurements, the cluster emission shows a large scale ellipticity, which could be an indicator of a past merger. The cluster has the lowest average temperature in the sample ( $\langle T$ map $\rangle \sim 2.3 \mathrm{keV}$, see Table 1 ) and one of the largest drops in entropy asymmetry from the centre to the outskirts (see Fig. 8). At about $20 \mathrm{kpc}$ from the central AGN we detect an enhancement in projected pressure of more than a factor of two compared to the enclosed ICM (see Fig. F.1). On larger scales the cluster shows a spiral structure in surface brightness, which is most likely caused by sloshing of gas due to a past merger (see Fig. E.1). In A 2052 all thermodynamic profiles are heavily influenced by the AGN feedback at the centre. Only the strongest feedback cases in our sample show a deviation from a radially decreasing pressure profile.

Abell 2146 is a major merger viewed almost edge-on. Russell et al. $(2010,2011,2012)$ detected two opposing shock fronts in the cluster and investigated transport processes in the ICM. They find the system to be less massive and thus colder than the Bullet Cluster merger (see also Fig. 10). Unlike in the Bullet Cluster, the secondary BCG in A2146 seems to be slightly lagging behind the shockfront (Canning et al. 2012).

The cluster shows the highest entropy perturbations in the sample (see Fig. 6). Asymmetries are especially high around the cluster centre that we choose in the smaller merging subcluster (see the cross in Fig. D.1). Like in 1E 0657-56, pressure fluctuations around the merging subcluster are rather low but increase at larger radii (see Fig. 8). The difference in $\mathrm{d} P / \mathrm{d} S$ between outer and inner radii is among the highest in the sample (see Fig. 8). The extreme entropy perturbations in A 2146 indicate the highest average Mach number in our sample (see Sect. 6.2), almost twice as high as in the Bullet Cluster. If the two merging systems have similar impact velocities, the lower temperature of A 2146 could cause the Mach number of the turbulence induced by the merger to be twice as high as in the Bullet Cluster.

Cygnus A and Hydra A are two similar systems with strong AGN feedback. Both sources have strong radio jets emerging from the central AGN causing complex structures in the X-ray emitting ICM around the nucleus (see e.g. McNamara et al. 2000; Smith et al. 2002, for Hydra A and Cygnus A respectively). Nulsen et al. $(2002,2005)$ found AGN feedback to influence the ICM on large scales in Hydra A.

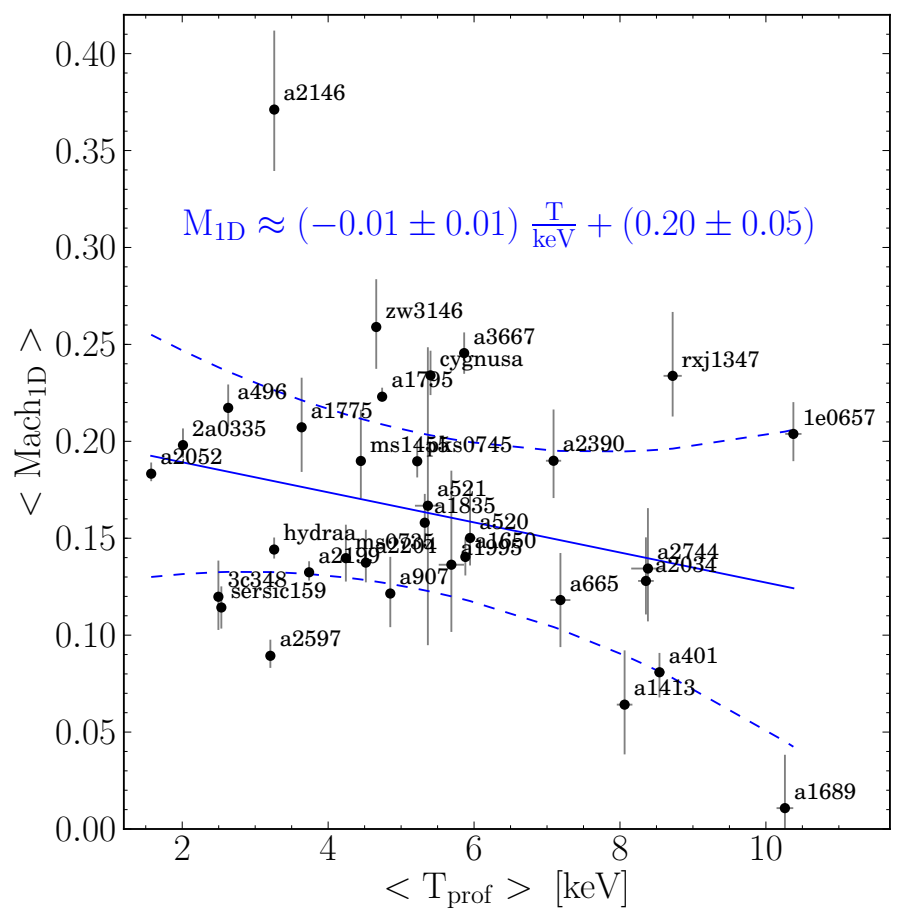

Fig. 10. Relation between the average cluster temperature $\langle T$ prof $\rangle$ and the fractional spread value of the dominating perturbation $(\mathrm{d} S$ or $\mathrm{d} P)$, which is proportional to the 1D Mach number. The solid and dashed lines represent the best linear correlation and its $1 \sigma$ scatter. Error bars are the statistical uncertainty from the MCMC measurements.

The unsharp-masked analysis of the surface-brightness images clearly shows the strong feedback structures around the central AGN (Fig. E.1). Average perturbations and temperature in Cygnus $\mathrm{A}$ are significantly higher than in Hydra A (see Figs. 6, 10). Cygnus A shows enhanced density and temperature at larger radii to the north-west (see Fig. D.1). Hydra A has a very asymmetric temperature distribution, which is mainly caused by continuous radial structures of colder gas extending from the centre (see Fig. D.1). The average metallicity of Cygnus A is significantly higher than for Hydra A (see Fig. 9). The thermodynamic profiles of Cygnus A are more disturbed, which would indicate a stronger or more recent AGN outburst.

Abell 2199 is a typical relaxed cluster with a cool core and AGN feedback structures at its centre (Markevitch et al. 1999; Johnstone et al. 2002). Nulsen et al. (2013) found various substructures in deep Chandra observations of the cluster, including evidence for a minor merger $\sim 400 \mathrm{Myr}$ ago. Sanders $\&$ Fabian (2006) found a weak isothermal shock ( $100 \mathrm{kpc}$ from the centre to the south-east) likely caused by the supersonic inflation of radio lobes by jets from the central AGN (3C 338).

Our maps indicate a weak temperature jump in the area where the shock has been detected (see Fig. D.1) and show a large scale asymmetry in the temperature distribution between north and south (also visible in the scatter of the radial profile, see Fig. F.1). The surface brightness unsharp-masked image shows some of the structures at the centre and a weak indication of the surface brightness jump due to the shock to the south-east. The perturbations in entropy, pressure, temperature, and density are in the average regime of our sample as expected for an overall relaxed system (see Figs. 6, 7). The radial profiles show a prominent discontinuity around $50 \mathrm{kpc}$ from the centre, related AGN feedback. Just outside this jump there is a region where 
the fit uncertainties are larger because of overlapping chip gaps of many observations, which could bias our results (see faint spurious linear structures in Fig. D.1).

Abell 496 is a relaxed cluster with relatively high metallicity around the cool core and non-uniform temperature distribution on large scales (Tamura et al. 2001; Tanaka et al. 2006; Ghizzardi et al. 2014). By comparing dedicated simulations of the cluster to deep Chandra observations, Roediger et al. (2012) concluded that the cluster was most likely perturbed by a merging subcluster $0.6-0.8$ Gyr ago.

The spiral surface brightness excess structure and the northern cold front is clearly visible in our new images of temperature and unsharp-masked count images (see Figs. D.1, E.1). The radial profiles and asymmetry measurements show relatively large spread in entropy (see Fig. F.1). Figures 6 and 7 show that the cluster has a large average temperature spread (as a result of the large scale asymmetry caused by the sloshing of colder gas from the centre) and thus also larger spread in entropy. A 496 has a flat pressure profile similar to the strongest merging systems in the sample. The average metallicity is the highest we measured.

PKS 0745-191 is a relaxed cluster at large scales out to the virial radius (George et al. 2009). Sanders et al. (2014) found AGN feedback and sloshing structures in deep Chandra observations.

The AGN feedback features are most prominent in the unsharp-masked image (Fig. E.1) and indications of weak asymmetry in temperature on large scales can be seen in Fig. D.1. The radial profiles of the cluster follow the expected trend for relaxed systems, but show remarkably low intrinsic scatter in pressure and relatively high scatter in entropy (see Fig. F.1). The scatter measurements of Sanders et al. (2014) are consistent with our method for $\mathrm{d} n, \mathrm{~d} T, \mathrm{~d} P$, and $\mathrm{d} S$. This is the only cluster in the sample where $n_{\mathrm{H}}$ was set as a free parameter in the spectral fits because the foreground column density of this system is known to vary significantly.

\section{Discussion}

The perturbation measurements for this cluster sample constrain the average Mach number of the systems. The sample covers a wide range of dynamic ICM states, providing insight into the influence of different perturbation events.

\subsection{Caveats of perturbation measurements}

It has been shown by Sanders et al. (2014) that in the PKS0745191 cluster projection effects in the measured parameters caused the calculated projected spread to be only about half of the real spread in the ICM. Therefore, we expect projection effects to strongly affect the absolute values of our spread parameters. Since the effect seems to be similar for all parameters in the PKS0745-191 system, the comparison of ratios between different spreads should not be affected. All asymmetry measurements are based on circular extraction regions, which means ellipticity of the cluster emission, as in PKS0745-191, adds to the spread values. Thus, strong ellipticity could also influence the measured ratios between perturbations. Sanders et al. (2014) showed for PKS0745-191 that different bin sizes within a certain range lead to consistent results in the spread analysis. However, if the bins are too large, as for some more distant clusters in our sample, we generally obtain larger absolute spread values (e.g. zw3146 in Fig. 6). Overall the comparison of spread measurements on maps with a $\mathrm{S} / \mathrm{N}$ of 25 and 50 were in good agreement.

In real clusters there are many factors influencing perturbation measurements. The clusters that lie outside the relations expected from simulations seem to be dominated by processes that have not been included in the simulations (like mergers, AGN feedback bubbles, shocks, or uplifted cold gas). Also projection effects in our measurements and the overall geometry of a system could cause deviations. We emphasise the parameters dS and dP are not independent, since they are derived from the independent fit parameters $\mathrm{T}$ and $\eta$ (see Sect. 2.2). Uncertainties in the spectral fits are larger at higher temperatures. The size of the error bars of individual measurements limit the sensitivity for finding additional spread in the MCMC calculations (see Sect. 3.1). In the case of Abell 1689, the absolute spread measurements are very low and we only obtain upper limits for this system, which is caused by very low fluctuations and high temperatures, lowering the sensitivity for detecting additional spread.

With the caveats described above, our measurements allow for a rough estimate of the average Mach number and thus turbulence trends in the ICM for a large sample of massive clusters of galaxies. Future simulations help to better quantify the influence of the above described caveats on the Mach number estimates.

\subsection{Relating perturbations to turbulence}

Recent high-resolution 3D hydrodynamic simulations by Gaspari et al. (2014) show that entropy and pressure drive the main perturbations in the ICM, depending on the Mach number in the medium. In the low Mach number case $\left(\operatorname{Mach}_{3 \mathrm{D}}<0.5\right)$ perturbations are mainly isobaric, implying

$\mathrm{d} P / P$ is negligible, $\mathrm{d} S / S \sim \operatorname{Mach}_{1 \mathrm{D}}$, and $\mathrm{d} n / n \sim|\mathrm{d} T / T|$.

For large Mach numbers $\left(\mathrm{Mach}_{3 \mathrm{D}}>0.5\right)$, perturbations shift to the adiabatic regime because turbulence becomes more violent, overcoming the cluster stratification, implying

$\mathrm{d} S / S$ becomes negligible, $\mathrm{d} P / P \sim \mathrm{Mach}_{1 \mathrm{D}}$, and $\mathrm{d} n / n \sim 1 /(\gamma-1) \mathrm{d} T / T \sim 1.5 \mathrm{~d} T / T$.

The measurements of the average perturbations in the sample suggest a mixture of the two states (see Figs. 6 and 7). We observed that entropy perturbations are slightly dominating pressure fluctuations (Fig. 6) and density fluctuations are comparable to temperature fluctuations with a possible tendency of slightly dominating $\mathrm{d} n$ (Fig. 7). Enhanced temperature asymmetry can be caused by displaced cold gas from the central region due to strong feedback like e.g. in Cygnus A or strong cold fronts, as observed in Abell 3667. Additional conduction in the ICM can weaken temperature perturbations (Gaspari et al. 2014).

Assuming entropy perturbations dominate in the sample, it follows that $\mathrm{d} S / S \sim \mathrm{Mach}_{1 \mathrm{D}}$. The $\mathrm{d} S / S$ value is related to the normalisation of the power spectrum, which is related to the peak of the power spectrum that occurs at large physical radii (low wavenumber $\mathrm{k}$ ), typically $\gtrsim 100 \mathrm{kpc}$ or $\gtrsim 0.1 r_{500}$. As an approximation, we used the overall average of perturbations $(M=1$, see Sect. 3.1) as a turbulence indicator since the assumption is that $\mathrm{dS}$ dominates does not hold in the outer regions $\gtrsim 100 \mathrm{kpc}$ of the clusters (see Sect. 4.2). The measurements on average suggest $\mathrm{Mach}_{1 \mathrm{D}} \approx 0.16 \pm 0.07$ (see Fig. 10).

Hydrodynamical simulations of galaxy clusters usually find the ratio between turbulence energy $E_{\text {turb }}$ and the thermal 
energy $E_{\text {therm }}$ to be $E_{\text {turb }} \approx 3-30 \% E_{\text {therm }}$, from relaxed to unrelaxed clusters (Norman \& Bryan 1999; Vazza et al. 2009; Lau et al. 2009; Vazza et al. 2011, 2012; Gaspari et al. 2012b; Miniati 2014; Schmidt et al. 2014). Since

$E_{\text {turb }}=0.5 \gamma(\gamma-1) \operatorname{Mach}_{3 \mathrm{D}}^{2} E_{\text {therm }} \simeq 0.56 \mathrm{Mach}_{3 \mathrm{D}}^{2} E_{\text {therm }}$,

where the adiabatic index $\gamma=\mathrm{C}_{\mathrm{P}} / \mathrm{C}_{\mathrm{V}}=5 / 3\left(\mathrm{C}_{\mathrm{P}}, \mathrm{C}_{\mathrm{V}}\right.$ heat capacity of the medium at constant pressure and volume, respectively), it follows that $\operatorname{Mach}_{3 \mathrm{D}} \simeq 0.23-0.73$ and thereby,

$\operatorname{Mach}_{1 \mathrm{D}}=\operatorname{Mach}_{3 \mathrm{D}} / \sqrt{3} \simeq 0.13-0.42$,

which is consistent with the measured average of $\mathrm{Mach}_{1 \mathrm{D}} \approx 0.16 \pm 0.07$. This would suggest that turbulence energy in this sample of clusters is on average about four per cent of the thermal energy in the systems. Figure 10 indicates a weak anti-correlation with average cluster temperature. In the best-fit linear regression $\mathrm{Mach}_{1 \mathrm{D}} \approx-(0.01 \pm 0.01)\left\langle T_{\text {prof }}\right\rangle / \mathrm{keV}+(0.20 \pm 0.05)$, the slope is consistent with zero within the errors. The significance of the slope was estimated using the re-sampling technique of Sect. 4.3 by investigating the distribution of fit functions in 1000 fits to random subsamples of half the size of the original. The absolute values used as Mach number indicator are subject to some uncertainty, such as projection and feedback effects that have not been taken into account in the comparison simulations (see Sect. 6.1).

\subsection{Difference between core and outskirts}

If the enhanced pressure perturbations in the outer regions of the clusters in this sample can be confirmed, this could be interpreted as a change in the thermodynamic state of the ICM from the centre to the outer regions. With increasing pressure perturbations dP we expect more turbulent, pressure-wave driven motions in the ICM.

This fits the expectation of less relaxed outer regions in clusters where the ICM is not yet virialised and still being accreted into the cluster potential (e.g. Lau et al. 2009). The change of the $\mathrm{dP} / \mathrm{dS}$ ratio could also be due to the dependence of the ratio on the probed scales (e.g. Gaspari \& Churazov 2013; Gaspari et al. 2014). In annuli at larger radii we probe larger scales of ICM fluctuations. A change in $\mathrm{d} P / \mathrm{d} S$ with scale would also show a radial dependence in the sample.

\subsection{ICM metallicity}

The metallicity of the ICM and its local distribution are of great interest when studying the processes that are enriching the ICM with heavier nuclei. These metals are thought to be mainly produced by supernova explosions in the galaxies (mainly in the brightest clusters galaxy, BCG) within the clusters and then transported into the ICM (see e.g. Böhringer \& Werner 2010; de Plaa et al. 2007).

The observed anti-correlation between average cluster temperature and metallicity (Fig. 9) could have many different causes. Sanders et al. (2004) found a similar relation between metallicity and temperature in the Perseus cluster. After testing for various systematic effects they found the correlation to be real, but no definite explanation for the effect has been found so far. Sloshing structures of colder gas have been found to coincide with higher metallicity (see e.g. Roediger et al. 2011), which could partially explain an anti-correlation.
The temperatures of the clusters in our sample are correlated to their redshift and the metallicity anti-correlates with redshift as well as temperature. Balestra et al. (2007) probed a sample of clusters in the redshift range $0.3<z<1.3$ and found significant evolution in metallicity between their higher and lower redshift clusters by more than a factor of two. This trend was confirmed by Maughan et al. (2008). Both studies also found a correlation between cluster temperature and metallicity. However, e.g. Baldi et al. (2012) found no significant evolution of abundance with redshift. It is not clear whether to expect any evolution within the narrow redshift range $(0.025 \leq z \leq 0.45)$ we probed. This corresponds to a time span of $\Delta t \approx 4 \mathrm{Gyr}$ in the standard cosmology assumed in this study.

In individual clusters there is a general trend of lower metallicity at larger radii (see also De Grandi \& Molendi 2001; Sanders et al. 2004; Leccardi \& Molendi 2008), which means the average metallicity is lower when we cover larger radial ranges for clusters at higher redshift. The metallicity drops from the centres $\left(Z_{\text {solar }} \approx 0.4-0.8\right)$ with typically lower temperature gas out to $\sim 300 \mathrm{kpc}$, where the profile flattens around a metallicity fraction of 0.2 solar. Uniform metallicity distribution at larger radii has been observed in great detail in the Perseus cluster (Werner et al. 2013).

The colder gas generally resides in the cluster centres, often in the vicinity of large $\mathrm{cD}$ galaxies that might cause enhanced enrichment. More massive (higher temperature) systems will have also undergone more mergers and generally have stronger AGN feedback, which contributes to the dilution of metals in the X-ray halo (e.g. Gaspari et al. 2011) and affects the average areaand error-weighted metallicity calculated in this study. Also, the multiphasedness of the hot ICM along the line of sight could influence the correlation as we typically probe larger volumes for higher temperatures. Multiphase gas has been found to bias the ICM metallicity measurements from X-ray spectra in some clusters (e.g. Panagoulia et al. 2013). Unresolved 2D structure of the ICM could bias the measured metallicity to higher values for systems with intermediate temperatures (see Rasia et al. 2008; Simionescu et al. 2009; Gastaldello et al. 2010). This study reduced multiphase effects by resolving the spatial structure and measuring the average metallicity of many spatial-spectral bins.

Another factor of influence could be that star formation efficiency decreases with cluster mass and temperature (e.g. Böhringer \& Werner 2010). There have been many studies in the infrared wavelength (e.g. Popesso et al. 2012, 2015) that show quenching of star formation in massive DM halos of galaxy clusters and groups. Our sample predominantly consists of relatively massive systems (see Table 1) at low to intermediate redshifts and in different stages of evolution. All metallicity measurements are based on a fixed solar abundance model (Anders \& Grevesse 1989).

\section{Summary and conclusions}

We presented a very large sample of detailed cluster maps with application to understand the thermodynamic processes in clusters of galaxies. The deep observations of the individual clusters helped to identify structures in the ICM caused by mergers or AGN feedback. By comparing perturbations in the sample with recent high-resolution simulations of perturbations in the ICM, we constrained the average 1D Mach number regime in the sample to $\mathrm{Mach}_{1 \mathrm{D}} \approx 0.16 \pm 0.07$ with some caveats (see Sect. 6). In comparison with simulations, this result would suggest $E_{\text {turb }} \approx 0.04 E_{\text {therm }}$ (see Sect. 6.2). By comparing perturbations in the central regions $(\lesssim 100 \mathrm{kpc})$ and in the outer regions 
$(\gtrsim 100 \mathrm{kpc})$, we found an indication for a change in the thermodynamic state from mainly isobaric to a more adiabatic regime.

In addition, the sample shows a tight correlation between the average cluster metallicity, average temperature, and redshift. The best-fit linear correlation between metallicity and redshift is $Z / Z_{\odot}=-(0.6 \pm 0.2) z+(0.36 \pm 0.04)$ and between metallicity and temperature the best fit is $Z / Z_{\odot}=-(1.0 \pm 0.7) T / 100 \mathrm{keV}+(0.34 \pm 0.06)$. The average metallicity of the sample is $Z \approx 0.3 \pm 0.1 Z_{\odot}$.

Future X-ray missions like Astro-H (Kitayama et al. 2014) and Athena (Nandra et al. 2013) will help to further investigate turbulent velocities and chemical enrichment in the ICM. The eROSITA observatory (Merloni et al. 2012) will detect a large $\mathrm{X}$-ray cluster sample for cosmological studies and our detailed cluster mapping can be used to make predictions on the scatter in scaling relations due to unresolved structures in temperature. To encourage further analysis based on this unique sample of cluster observations, all maps and asymmetry measurements used in this study are made available publicly in electronic form.

Acknowledgements. We thank the anonymous referee for constructive com ments that helped to improve the clarity of the paper. We thank J. Buchner for helpful discussions. This research has made use of data obtained from the Chandra Data Archive and the Chandra Source Catalog, and software provided by the Chandra X-ray Center (CXC) in the application packages CIAO, ChIPS, and Sherpa. This research has made use of NASA's Astrophysics Data System. This research has made use of the VizieR catalogue access tool, CDS, Strasbourg, France. This research has made use of SAOImage DS9, developed by Smithsonian Astrophysical Observatory. This research has made use of data and software provided by the High Energy Astrophysics Science Archive Research Center (HEASARC), which is a service of the Astrophysics Science Division at NASA/GSFC and the High Energy Astrophysics Division of the Smithsonian Astrophysical Observatory. This research has made use of the SIMBAD database, operated at CDS, Strasbourg, France. This research has made use of the tools Veusz, the matplotlib library for Python, and TOPCAT. M.G. is supported by the National Aeronautics and Space Administration through Einstein Postdoctoral Fellowship Award Number PF-160137 issued by the Chandra X-ray Observatory Center, which is operated by the Smithsonian Astrophysical Observatory for and on behalf of the National Aeronautics Space Administration under contract NAS8-03060.

\section{References}

Allen, S. W., Schmidt, R. W., \& Fabian, A. C. 2002, MNRAS, 334, L11 Allen, S. W., Schmidt, R. W., Ebeling, H., Fabian, A. C., \& van Speybroeck, L. 2004, MNRAS, 353, 457

Allen, S. W., Rapetti, D. A., Schmidt, R. W., et al. 2008, MNRAS, 383, 879 Anders, E., \& Grevesse, N. 1989, Geochim. Cosmochim. Acta, 53, 197 Arnaud, K. A. 1996, in Cosmic Abundances, eds. S. S. Holt, \& G. Sonneborn, ASP Conf. Ser., 99, 409

Baldi, A., Ettori, S., Molendi, S., Balestra, I., et al. 2012, A\&A, 537, A142

Balestra, I., Tozzi, P., Ettori, S., et al. 2007, A\&A, 462, 429

Blanton, E. L., Sarazin, C. L., McNamara, B. R., \& Wise, M. W. 2001, ApJ, 558, L15

Blanton, E. L., Sarazin, C. L., \& McNamara, B. R. 2003, ApJ, 585, 227

Blanton, E. L., Randall, S. W., Clarke, T. E., et al. 2011, ApJ, 737, 99

Böhringer, H., \& Werner, N. 2010, A\&ARv, 18, 127

Böhringer, H., Voges, W., Huchra, J. P., et al. 2000, ApJS, 129, 435

Böhringer, H., Schuecker, P., Guzzo, L., et al. 2004, A\&A, 425, 367

Bradač, M., Clowe, D., Gonzalez, A. H., et al. 2006, ApJ, 652, 937

Broadhurst, T. J., Taylor, A. N., \& Peacock, J. A. 1995, ApJ, 438, 49

Canning, R. E. A., Russell, H. R., Hatch, N. A., et al. 2012, MNRAS, 420, 2956 Cash, W. 1979, ApJ, 228, 939

Clowe, D., Gonzalez, A., \& Markevitch, M. 2004, ApJ, 604, 596

Clowe, D., Bradač, M., Gonzalez, A. H., et al. 2006, ApJ, 648, L109

De Grandi, S., \& Molendi, S. 2001, ApJ, 551, 153

de Plaa, J., Werner, N., Bleeker, J. A. M., et al. 2007, A\&A, 465, 345

Dennis, T. J., \& Chandran, B. D. G. 2005, ApJ, 622, 205

Dolag, K., Schindler, S., Govoni, F., \& Feretti, L. 2001, A\&A, 378, 777

Dolag, K., Vazza, F., Brunetti, G., \& Tormen, G. 2005, MNRAS, 364, 753

Ebeling, H., Mullis, C. R., \& Tully, R. B. 2002, ApJ, 580, 774

Ehlert, S., McDonald, M., David, L. P., Miller, E. D., \& Bautz, M. W. 2015, ApJ, 799,174
Ettori, S., Fabian, A. C., Allen, S. W., \& Johnstone, R. M. 2002, MNRAS, 331, 635

Fabian, A. C. 1994, ARA\&A, 32, 277

Fabian, A. C., Sanders, J. S., Ettori, S., et al. 2000, MNRAS, 318, L65

Fabian, A. C., Sanders, J. S., Ettori, S., et al. 2001, MNRAS, 321, L33

Fabian, A. C., Sanders, J. S., Taylor, G. B., et al. 2006, MNRAS, 366, 417

Foreman-Mackey, D., Hogg, D. W., Lang, D., \& Goodman, J. 2013, PASP, 125 , 306

Forman, W., Jones, C., Churazov, E., et al. 2007, ApJ, 665, 1057

Foster, A. R., Ji, L., Smith, R. K., \& Brickhouse, N. S. 2012, ApJ, 756, 128

Fruscione, A., McDowell, J. C., Allen, G. E., et al. 2006, in SPIE Conf. Ser., 6270,1

Garmire, G. P., Bautz, M. W., Ford, P. G., Nousek, J. A., \& Ricker, Jr., G. R. 2003, in X-Ray and Gamma-Ray Telescopes and Instruments for Astronomy, eds. J. E. Truemper, \& H. D. Tananbaum, SPIE Conf. Ser., 4851, 28

Gaspari, M., \& Churazov, E. 2013, A\&A, 559, A78

Gaspari, M., Melioli, C., Brighenti, F., \& D'Ercole, A. 2011, MNRAS, 411, 349 Gaspari, M., Brighenti, F., \& Temi, P. 2012a, MNRAS, 424, 190 Gaspari, M., Ruszkowski, M., \& Sharma, P. 2012b, ApJ, 746, 94

Gaspari, M., Churazov, E., Nagai, D., \& Lau, E. T., \& Zhuravleva, I. 2014, A\&A, 569, A67

Gastaldello, F., Ettori, S., Balestra, I., et al. 2010, A\&A, 522, A34

George, M. R., Fabian, A. C., Sanders, J. S., Young, A. J., \& Russell, H. R. 2009, MNRAS, 395, 657

Ghizzardi, S., De Grandi, S., \& Molendi, S. 2014, A\&A, 570, A117

Govoni, F., Markevitch, M., Vikhlinin, A., et al. 2004, ApJ, 605, 695

Graessle, D. E., Evans, I. N., Glotfelty, K., et al. 2007, Chandra News, 14, 33

Hogg, D. W., Bovy, J., \& Lang, D. 2010, ArXiv e-prints [arXiv: 1008 .4686]

Johnstone, R. M., Allen, S. W., Fabian, A. C., \& Sanders, J. S. 2002, MNRAS, 336, 299

Kaiser, N., Squires, G., \& Broadhurst, T. 1995, ApJ, 449, 460

Kalberla, P. M. W., Burton, W. B., Hartmann, D., et al. 2005, A\&A, 440, 775

Kitayama, T., Bautz, M., Markevitch, M., et al. 2014, ArXiv e-prints [arXiv: 1412.1176]

Lau, E. T., Kravtsov, A. V., \& Nagai, D. 2009, ApJ, 705, 1129

Leccardi, A., \& Molendi, S. 2008, A\&A, 487, 461

Machado, R. E. G., \& Lima Neto, G. B. 2015, MNRAS, 447, 2915

Mahdavi, A., Hoekstra, H., Babul, A., et al. 2013, ApJ, 767, 116

Markevitch, M., Vikhlinin, A., Forman, W. R., \& Sarazin, C. L. 1999, ApJ, 527 545

Markevitch, M., Ponman, T. J., Nulsen, P. E. J., et al. 2000, ApJ, 541, 542

Markevitch, M., Vikhlinin, A., \& Mazzotta, P. 2001, ApJ, 562, L153

Markevitch, M., Gonzalez, A. H., David, L., et al. 2002, ApJ, 567, L27

Markevitch, M., Bautz, M. W., Biller, B., et al. 2003, ApJ, 583, 70

Markevitch, M., Gonzalez, A. H., Clowe, D., et al. 2004, ApJ, 606, 819

Maughan, B. J., Jones, C., Forman, W., \& Van Speybroe ck, L. 2008, ApJS, 174 117

McDonald, M., Veilleux, S., Rupke, D. S. N., Mushotzky, R., \& Reynolds, C. 2011, ApJ, 734, 95

McNamara, B. R., Wise, M., Nulsen, P. E. J., et al. 2000, ApJ, 534, L135

Merloni, A., Predehl, P., Becker, W., et al. 2012, ArXiv e-prints [arXiv: 1209.3114]

Miniati, F. 2014, ApJ, 782, 21

Nandra, K., Barret, D., Barcons, X., et al. 2013, ArXiv e-prints [arXiv: 1306.2307]

Nelson, K., Lau, E. T., \& Nagai, D. 2014, ApJ, 792, 25

Norman, M. L., \& Bryan, G. L. 1999, in Lecture Notes in Physics, The Radio Galaxy Messier 87, eds. H.-J. Röser, \& K. Meisenheimer (Berlin Springer Verlag), 530, 106

Nulsen, P. E. J., David, L. P., McNamara, B. R., et al. 2002, ApJ, 568, 163

Nulsen, P. E. J., McNamara, B. R., Wise, M. W., \& David, L. P. 2005, ApJ, 628, 629

Nulsen, P. E. J., Li, Z., Forman, W. R., et al. 2013, ApJ, 775, 117

Oegerle, W. R., \& Hill, J. M. 1994, AJ, 107, 857

Panagoulia, E. K., Fabian, A. C., \& Sanders, J. S. 2013, MNRAS, 433, 3290

Pinto, C., Sanders, J. S., Werner, N., et al. 2015, A\&A, 575, A38

Popesso, P., Biviano, A., Rodighiero, G., et al. 2012, A\&A, 537, A58

Popesso, P., Biviano, A., Finoguenov, A., et al. 2015, A\&A, 574, A105

Randall, S. W., Markevitch, M., Clowe, D., Gonzalez, A. H., \& Bradač, M. 2008,

ApJ, 679, 1173

Rasia, E., Mazzotta, P., Bourdin, H., et al. 2008, ApJ, 674, 728

Reiprich, T. H., \& Böhringer, H. 2002, ApJ, 567, 716

Rines, K., Geller, M. J., Diaferio, A., \& Kurtz, M. J. 2013, ApJ, 767, 15

Roediger, E., Brüggen, M., Simionescu, A., et al. 2011, MNRAS, 413, 2057

Roediger, E., Lovisari, L., Dupke, R., et al. 2012, MNRAS, 420, 3632

Russell, H. R., Sanders, J. S., Fabian, A. C., et al. 2010, MNRAS, 406, 1721

Russell, H. R., van Weeren, R. J., Edge, A. C., et al. 2011, MNRAS, 417, L1

Russell, H. R., McNamara, B. R., Sanders, J. S., et al. 2012, MNRAS, 423, 236 
A\&A 585, A130 (2016)

Ruszkowski, M. \& Oh, S. P. 2010, ApJ, 713, 1332

Sanders, J. S. 2006, MNRAS, 371, 829

Sanders, J. S., \& Fabian, A. C. 2006, MNRAS, 371, L65

Sanders, J. S., \& Fabian, A. C. 2013, MNRAS, 429, 2727

Sanders, J. S., Fabian, A. C., Allen, S. W., \& Schmidt, R. W. 2004, MNRAS, 349,952

Sanders, J. S., Fabian, A. C., \& Taylor, G. B. 2005, MNRAS, 356, 1022

Sanders, J. S., Fabian, A. C., \& Smith, R. K. 2011, MNRAS, 410, 1797

Sanders, J. S., Fabian, A. C., Hlavacek-Larrondo, J., et al. 2014, MNRAS, 444 , 1497

Sarazin, C. L. 1986, Rev. Mod. Phys., 58, 1

Schmidt, W., Almgren, A. S., Braun, H., et al. 2014, MNRAS, 440, 3051

Schuecker, P., Finoguenov, A., Miniati, F., Böh ringer, H., \& Briel, U. G. 2004, A\&A, 426, 387

Simionescu, A., Werner, N., Böhringer, H., et al. 2009, A\&A, 493, 409

Smith, D. A., Wilson, A. S., Arnaud, K. A., Terashi ma, Y., \& Young, A. J. 2002, ApJ, 565, 195

Springel, V., White, S. D. M., Jenkins, A., et al. 2005, Nature, 435, 629
Tamura, T., Bleeker, J. A. M., Kaastra, J. S., Ferrigno, C., \& Molendi, S. 2001, A\&A, 379, 107

Tanaka, T., Kunieda, H., Hudaverdi, M., Furuzawa, A., \& Tawara, Y. 2006, PASJ, 58,703

Truemper, J. 1982, Adv. Space Res., 2, 241

Vazza, F., Brunetti, G., Kritsuk, A., Wagner, R., Gheller, C., \& Norman, M. 2009, A\&A, 504, 33

Vazza, F., Brunetti, G., Gheller, C., Brunino, R., \& Brüggen, M. 2011, A\&A, 529, A17

Vazza, F., Roediger, E., \& Brüggen, M. 2012, A\&A, 544, A103

Vikhlinin, A., Burenin, R. A., Ebeling, H., et al. 2009a, ApJ, 692, 1033

Vikhlinin, A., Kravtsov, A. V., Burenin, R. A., et al. 2009b, ApJ, 692, 1060

Voit, G. M. 2005, Rev. Mod. Phys., 77, 207

Walker, S. A., Fabian, A. C., \& Kosec, P. 2014, MNRAS, 445, 3444

Werner, N., Urban, O., Simionescu, A., \& Allen, S. W. . 2013, Nature, 502, 656

Zhang, Y.-Y., Finoguenov, A., Böhringer, H., et al. 2008, A\&A, 482, 451

Zhang, Y.-Y., Andernach, H., Caretta, C. A., et al. 2011, A\&A, 526, A105

Zhuravleva, I., Churazov, E., Schekochihin, A. A., et al. 2014, Nature, 515, 85 


\section{Appendix A: Cluster sample}

Table A.1. Chandra cluster sample (CIZA clusters).

\begin{tabular}{lrrrrrrr}
\hline \hline Cluster $^{a}$ & $\begin{array}{l}\text { Exp. }^{b} \\
{[\mathrm{ks}]}\end{array}$ & $\begin{array}{l}\text { RA (J2000) } \\
{[\mathrm{deg}]}\end{array}$ & $\begin{array}{l}\text { DEC (J2000) } \\
{[\mathrm{deg}]}\end{array}$ & $\begin{array}{l}\text { Flux } \\
{\left[10^{-15} \mathrm{~W} / \mathrm{m}^{2}\right]}\end{array}$ & & Obj. ID (CIZA) & \multicolumn{1}{l}{$\begin{array}{l}L_{\mathrm{X}}{ }^{d} \\
{\left[10^{37} \mathrm{~W}\right]}\end{array}$} \\
\hline CYGNUS A & 232 & 299.877 & 40.741 & 52.82 & 0.0561 & $\mathrm{~J} 1959.5+4044$ & 7.08 \\
PKS 0745-191 & 174 & 116.883 & -19.290 & 45.73 & 0.1028 & J0747.5-1917 & 20.36 \\
\hline
\end{tabular}

Notes. ${ }^{(a)}$ Most commonly used name of cluster or central object. ${ }^{(b)}$ Combined ACIS-S/-I exposure after excluding times of high background.

${ }^{(c)}$ ROSAT $0.1-2.4 \mathrm{keV}$ X-ray flux in $10^{-15} \mathrm{~W} / \mathrm{m}^{2}$. ${ }^{(d)}$ ROSAT $0.1-2.4 \mathrm{keV}$ X-ray luminosity in $10^{37} \mathrm{~W}$ (CIZA column: LX).

Table A.2. Chandra cluster sample (NORAS clusters).

\begin{tabular}{|c|c|c|c|c|c|c|c|}
\hline Cluster $^{a}$ & $\begin{array}{l}\text { Exp. }^{b} \\
{[\mathrm{ks}]}\end{array}$ & $\begin{array}{r}\text { RA (J2000) } \\
\text { [deg] }\end{array}$ & $\begin{array}{r}\operatorname{Dec}(\mathrm{J} 2000) \\
{[\mathrm{deg}]}\end{array}$ & $\begin{array}{r}\text { Flux }^{c} \\
{\left[10^{-15} \mathrm{~W} / \mathrm{m}^{2}\right]}\end{array}$ & $z$ & Obj. ID (NORAS) & $\begin{array}{r}L_{\mathrm{X}}{ }^{d} \\
{\left[10^{37} \mathrm{~W}\right]}\end{array}$ \\
\hline A 2052 & 651 & 229.182 & 7.013 & 47.94 & 0.0353 & RXC J1516.7+0701 & 2.58 \\
\hline A 1775 & 99 & 205.448 & 26.352 & 12.58 & 0.0724 & RXC J1341.8+2622 & 2.83 \\
\hline A 2199 & 156 & 247.188 & 39.553 & 97.92 & 0.0299 & RXC J1628.6+3932 & 3.77 \\
\hline 2A $0335+096$ & 102 & 54.665 & 10.007 & 80.91 & 0.0347 & RXC J0338.6+0958 & 4.21 \\
\hline 3C348 (HERCULES A) & 112 & 252.778 & 4.985 & 5.39 & 0.154 & RXC J1651.1+0459 & 5.49 \\
\hline A 2034 & 255 & 227.532 & 33.515 & 11.94 & 0.113 & RXC J1510.1+3330 & 6.49 \\
\hline MS0735.6+7421 & 520 & 115.421 & 74.266 & 4.06 & 0.2149 & RXC J0741.7+7414 & 7.94 \\
\hline A 2146 & 418 & 239.006 & 66.352 & 3.99 & 0.2339 & RXC J1556.1+6621 & 9.31 \\
\hline A 1795 & 958 & 207.221 & 26.596 & 59.29 & 0.0622 & RXC J1348.8+2635 & 9.93 \\
\hline A 1413 & 136 & 178.769 & 23.369 & 12.61 & 0.1427 & RXC J1155.3+2324 & 10.91 \\
\hline A 401 & 163 & 44.727 & 13.579 & 50.29 & 0.0739 & RXC J0258.9+1334 & 11.76 \\
\hline A 1995 & 100 & 223.168 & 58.049 & 3.18 & 0.3179 & RXC J1452.9+5802 & 13.42 \\
\hline MS $1455.0+2232$ & 108 & 224.253 & 22.33 & 4.89 & 0.2579 & RXC J1457.2+2220 & 13.73 \\
\hline A 520 & 527 & 73.546 & 2.977 & 8.33 & 0.203 & RXC J0454.1+0255 & 14.52 \\
\hline A 665 & 140 & 127.637 & 65.89 & 11.18 & 0.1818 & RXC J0830.9+6551 & 15.69 \\
\hline A 2204 & 97 & 248.186 & 5.557 & 24.11 & 0.1514 & RXC J1632.7+0534 & 23.43 \\
\hline A 2390 & 110 & 328.403 & 17.683 & 11.01 & 0.2329 & RXC J2153.5+1741 & 25.15 \\
\hline ZW 3146 & 84 & 155.906 & 4.167 & 8.77 & 0.285 & RXC J1023.6+0411 & 29.91 \\
\hline A 1835 & 223 & 210.271 & 2.895 & 12.12 & 0.2528 & RXC J1401.0+0252 & 32.56 \\
\hline
\end{tabular}

Notes. ${ }^{(a)}$ Most commonly used name of cluster or central object. ${ }^{(b)}$ Combined ACIS-S/-I exposure after excluding times of high background.

${ }^{(c)}$ ROSAT $0.1-2.4 \mathrm{keV}$ X-ray flux in $10^{-15} \mathrm{~W} / \mathrm{m}^{2}$. ${ }^{(d)}$ ROSAT $0.1-2.4 \mathrm{keV}$ X-ray luminosity in $10^{37} \mathrm{~W}$ (NORAS column: LX).

Table A.3. Chandra cluster sample (REFLEX clusters).

\begin{tabular}{|c|c|c|c|c|c|c|c|}
\hline Cluster $^{a}$ & $\begin{array}{l}\text { Exp. }^{b} \\
{[\mathrm{ks}]}\end{array}$ & $\begin{array}{r}\text { RA (J2000) } \\
\text { [deg] }\end{array}$ & $\begin{array}{r}\text { Dec (J2000) } \\
\text { [deg] }\end{array}$ & $\begin{array}{r}\text { Flux }^{c} \\
{\left[10^{-15} \mathrm{~W} / \mathrm{m}^{2}\right]}\end{array}$ & $z$ & Obj. ID (REFLEX) & $\begin{array}{r}L_{\mathrm{X}}{ }^{d} \\
{\left[10^{37} \mathrm{~W}\right]}\end{array}$ \\
\hline SERSIC 159-03 & 106 & 348.515 & -42.713 & 23.412 & 0.0564 & $\mathrm{~J} 2313.9-4244$ & 3.74 \\
\hline A 496 & 88 & 68.403 & -13.25 & 72.075 & 0.0326 & J0433.6-1315 & 3.89 \\
\hline HYDRA A & 224 & 139.527 & -12.092 & 39.461 & 0.0539 & J0918.1-1205 & 5.61 \\
\hline A 1650 & 229 & 194.664 & -1.781 & 20.909 & 0.0845 & $\mathrm{~J} 1258.6-0145$ & 6.99 \\
\hline A 2597 & 146 & 351.337 & -12.136 & 20.558 & 0.0852 & $\mathrm{~J} 2325.3-1207$ & 7.22 \\
\hline A 3667 & 528 & 303.211 & -56.855 & 70.892 & 0.0556 & J2012.5-5649 & 10.02 \\
\hline A 907 & 103 & 149.528 & -11.086 & 7.833 & 0.1669 & J0958.3-1103 & 10.13 \\
\hline A 521 & 165 & 73.558 & -10.273 & 4.944 & 0.2475 & J0454.1-1014 & 12.97 \\
\hline A 2744 & 124 & 3.586 & -30.352 & 4.964 & 0.3066 & $\mathrm{~J} 0014.3-3023$ & 19.79 \\
\hline A 1689 & 197 & 197.808 & -1.337 & 15.332 & 0.1832 & $\mathrm{~J} 1311.4-0120$ & 23.59 \\
\hline 1E 0657-56 & 566 & 104.751 & -55.904 & 9.079 & 0.2965 & J0658.5-5556 & 35.55 \\
\hline RX J1347-114 & 232 & 206.889 & -11.734 & 6.468 & 0.4516 & J1347.5-1144 & 63.43 \\
\hline
\end{tabular}

Notes. ${ }^{(a)}$ Most commonly used name of cluster or central object. ${ }^{(b)}$ Combined ACIS-S/-I exposure after excluding times of high background.

${ }^{(c)}$ ROSAT $0.1-2.4 \mathrm{keV}$ X-ray flux in $10^{-15} \mathrm{~W} / \mathrm{m}^{2}$. ${ }^{(d)}$ ROSAT $0.1-2.4 \mathrm{keV}$ X-ray luminosity in $10^{37} \mathrm{~W}$ (REFLEX column: LumCor, $h=0.5$ ). 


\section{Appendix B: Chandra datasets}

Table B.1. Chandra datasets used in this study.

\begin{tabular}{|c|c|}
\hline Cluster $^{a}$ & Chandra $\mathrm{ObsID}^{b}$ \\
\hline $1 \mathrm{e} 0657$ & $554,3184,4984,4985,4986,5355,5356,5357,5358,5361$ \\
\hline $2 \mathrm{a} 0335$ & $919,7939,9792$ \\
\hline $3 \mathrm{c} 348$ & $1625,5796,6257$ \\
\hline a1413 & $537,1661,5002,5003,7696$ \\
\hline a1650 & $4178,5822,5823,6356,6357,6358,7242,7691$ \\
\hline a1689 & $540,1663,5004,6930,7289,7701$ \\
\hline a1775 & 12891,13510 \\
\hline a1795 & $\begin{array}{l}\text { 493, 494, 3666, 5286, 5287, 5288, 5289, 5290, 6159, 6160, 6161, 6162, 6163, } \\
10432,10433,10898,10899,10900,10901,12026,12027,12028,12029,13106,13107, \\
13108,13109,13110,13111,13112,13113,13412,13413,13414,13415,13416,13417, \\
14268,14269,14270,14271,14272,14273,14274,14275,15485,15486,15487,15488,15489, \\
15490,15491,15492,16432,16433,16434,16435,16436,16437,16438,16439,16465,16466, \\
16467,16468,16469,16470,16471,16472\end{array}$ \\
\hline a1835 & $495,496,6880,6881,7370$ \\
\hline a1995 & $906,7021,7713$ \\
\hline a2034 & $2204,7695,12885,12886,13192,13193$ \\
\hline a2052 & $890,5807,10477,10478,10479,10480,10879,10914,10915,10916,10917$ \\
\hline $\mathrm{a} 2146$ & $10464,10888,12245,12246,12247,13020,13021,13023,13120,13138$ \\
\hline a2199 & $497,498,10748,10803,10804,10805$ \\
\hline a2204 & $499,6104,7940$ \\
\hline a2390 & $500,501,4193$ \\
\hline a2597 & $922,6934,7329,15144$ \\
\hline a2744 & $2212,7712,7915,8477,8557$ \\
\hline a3667 & $513,889,5751,5752,5753,6292,6295,6296,7686$ \\
\hline a401 & $518,2309,14024$ \\
\hline a496 & $931,3361,4976$ \\
\hline a520 & $528,4215,7703,9424,9425,9426,9430$ \\
\hline a521 & $430,901,12880,13190$ \\
\hline a665 & $531,3586,7700,12286,13201,15148$ \\
\hline a907 & $535,3185,3205$ \\
\hline cygnusa & $360,5830,5831,6225,6226,6228,6229,6250,6252$ \\
\hline hydraa & $575,576,4969,4970$ \\
\hline $\mathrm{ms} 0735$ & 4197, 10468, 10469, 10470, 10471, 10822, 10918, 10922 \\
\hline ms1455 & $543,4192,7709$ \\
\hline pks0745 & $508,2427,6103,7694,12881$ \\
\hline $\operatorname{rxj} 1347$ & $506,507,3592,13516,13999,14407$ \\
\hline sersic159 & 1668,11758 \\
\hline Zw3146 & 909,9371 \\
\hline
\end{tabular}

Notes. ${ }^{(a)}$ Abbreviated cluster name. ${ }^{(b)}$ List of Chandra observations used in this study (indicated by their ObsID, observation identification number). 
F. Hofmann et al.: Thermodynamic perturbations in galaxy clusters

\section{Appendix C: Perturbation table}

Table C.1. Measured fractional perturbations.

\begin{tabular}{|c|c|c|c|c|}
\hline Cluster $^{a}$ & $\begin{array}{c}\mathrm{d} P^{b} \\
\text { [per cent] }\end{array}$ & $\begin{array}{c}\mathrm{d} S^{b} \\
\text { [per cent] }\end{array}$ & $\begin{array}{r}\mathrm{d} T^{b} \\
\text { [per cent] }\end{array}$ & $\begin{array}{r}\mathrm{d} n^{b} \\
\text { [per cent] }\end{array}$ \\
\hline $1 \mathrm{e} 0657$ & $20.4_{-1.4}^{+1.6}$ & $17.3_{-15}^{+2.1}$ & $8.8_{-1.4}^{+1.5}$ & $20.9_{-0.9}^{+1.2}$ \\
\hline $2 \mathrm{a} 0335$ & $11.7_{-0.5}^{+0.6}$ & $19.8_{-0.6}^{+0.9}$ & $9.3_{-0.4}^{+0.5}$ & $13.5_{-0.4}^{+0.6}$ \\
\hline $3 \mathrm{c} 348$ & $1.3_{-1.3}^{+2.9}$ & $12.0_{-1.7}^{+1.9}$ & $3.6_{-2.8}^{+2.0}$ & $8.5_{-0.6}^{+0.9}$ \\
\hline a1413 & $5.4_{-4.0}^{+1.9}$ & $6.4_{-2.6}^{+2.8}$ & $0.3_{-0.3}^{+2.6}$ & $8.7_{-0.7}^{+1.0}$ \\
\hline a1650 & $5.5_{-1.0}^{+0.9}$ & $14.0_{-1.0}^{+1.0}$ & $4.0_{-1.0}^{+1.0}$ & $10.0_{-0.5}^{+0.7}$ \\
\hline a1689 & $0.6_{-0.6}^{+2.7}$ & $1.1_{-1.1}^{+2.8}$ & $0.8_{-0.8}^{+2.1}$ & $3.8_{-0.2}^{+0.4}$ \\
\hline a1775 & $0.6_{-0.6}^{+3.5}$ & $20.7_{-2.3}^{+2.6}$ & $12.6_{-2.1}^{+1.9}$ & $8.0_{-0.8}^{+0.8}$ \\
\hline a1795 & $5.3_{-0.2}^{+0.2}$ & $22.3_{-0.2}^{+0.5}$ & $11.7_{-0.1}^{+0.4}$ & $12.4_{-0.1}^{+0.3}$ \\
\hline a1835 & $6.0_{-1.1}^{+1.1}$ & $15.8_{-1.1}^{+1.5}$ & $9.3_{-0.8}^{+1.0}$ & $8.8_{-0.5}^{-0.1}$ \\
\hline a1995 & $5.7_{-57}^{-1.1 .5}$ & $13.6_{-35}^{+4.9}$ & $11.4_{-5.8}^{+3.8}$ & $7.0_{-0.9}^{-1.6}$ \\
\hline a2034 & $12.8_{-1.7}^{+2.2}$ & $6.5_{-2.4}^{+2.1}$ & $6.1_{-3.4}^{+1.6}$ & $9.1_{-0.7}^{+1.0}$ \\
\hline a2052 & $7.5_{-0.2}^{+0.2}$ & $18.3_{-0.4}^{+0.6}$ & $11.6_{-0.2}^{+0.4}$ & $10.1_{-0.2}^{+0.3}$ \\
\hline a2146 & $20.3_{-1.9}^{+1.9}$ & $37.1_{-3.2}^{+4.4}$ & $12.2_{-1.4}^{+1.7}$ & $24.3_{-1.5}^{+2.7}$ \\
\hline a2199 & $7.6_{-0.3}^{+0.4}$ & $13.2_{-0.4}^{+0.6}$ & $7.3_{-0.3}^{+0.4}$ & $8.6_{-0.3}^{+0.3}$ \\
\hline a2204 & $6.7_{-1.3}^{-1.3}$ & $13.7_{-1.0}^{+1.4}$ & $8.7_{-1.0}^{-1 . .1}$ & $7.6_{-0.5}^{-0.5}$ \\
\hline a2390 & $11.4_{-1.8}^{+2.0}$ & $19.0_{-1.9}^{+2.6}$ & $12.4_{-2.0}^{+2.1}$ & $11.2_{-0.8}^{+1.1}$ \\
\hline a2597 & $8.1_{-0.6}^{+0.8}$ & $8.9_{-0.6}^{+0.8}$ & $5.5_{-0.6}^{+0.6}$ & $7.2_{-0.4}^{+0.5}$ \\
\hline a2744 & $9.8_{-9.8}^{+4.0}$ & $13.4_{-2.7}^{+3.0}$ & $\begin{array}{l}7.6_{-7.06}^{+3.5} \\
\text {. }\end{array}$ & $15.1_{-1.1}^{+1.4}$ \\
\hline a3667 & $24.6_{-1.1}^{+1.1}$ & $12.4_{-0.5}^{+0.7}$ & $16.1_{-0.7}^{+0.9}$ & $11.4_{-0.4}^{+0.5}$ \\
\hline a401 & $6.1_{-1.5}^{+1.3}$ & $8.1_{-1.3}^{+1.0}$ & $2.8_{-2.8}^{+1.3}$ & $7.5_{-0.3}^{+0.5}$ \\
\hline a496 & $9.0_{-0.6}^{+0.6}$ & $21.7_{-1.0}^{+1.2}$ & $14.0_{-0.6}^{+0.9}$ & $8.9_{-0.4}^{+0.5}$ \\
\hline a520 & $12.6_{-1.8}^{+2.0}$ & $15.0_{-1.4}^{+2.6}$ & $7.6_{-1.7}^{+1.9}$ & $11.5_{-0.9}^{+1.4}$ \\
\hline a521 & $12.1_{-7.8}^{-5.8}$ & $16.7_{-7.2}^{-8.4}$ & $10.0_{-8.0}^{+6.1 .1}$ & $10.7_{-1.4}^{+2.3}$ \\
\hline a665 & $11.8_{-2.4}^{+2.4}$ & $8.2_{-4.6}^{+2.6}$ & $6.5_{-3.9}^{+2.6}$ & $11.0_{-0.8}^{+0.9}$ \\
\hline a907 & $0.2_{-0.2}^{+2.4}$ & $12.1_{-1.7}^{+1.9}$ & $1.7_{-1.7}^{+2.9}$ & $9.5_{-0.6}^{+0.9}$ \\
\hline cygnusa & $18.2_{-0.8}^{+1.0}$ & $23.4_{-1.0}^{+1.3}$ & $14.1_{-0.6}^{+0.1}$ & $27.3_{-0.9}^{+0.0}$ \\
\hline hydraa & $9.8_{-0.4}^{+0.0}$ & $14.4_{-0.5}^{+0.6}$ & $9.7_{-0.4}^{-0.0}$ & $7.8_{-0.2}^{-0.3}$ \\
\hline $\mathrm{ms} 0735$ & $10.6_{-0.9}^{-0.5}$ & $14.0_{-12}^{+0.5}$ & $7.8_{-0.8}^{-1.3}$ & $10.3_{-07}^{+0.2}$ \\
\hline $\mathrm{ms} 1455$ & $4.1_{-3.5}^{+2.0}$ & $19.0_{-1.9}^{+2.6}$ & $9.0_{-1.5}^{+2.0}$ & $11.1_{-0.8}^{+1.0}$ \\
\hline pks0745 & $3.4_{-1.9}^{+1.0}$ & $19.0_{-0.8}^{+1.0}$ & $9.4_{-0.6}^{+0.8}$ & $11.6_{-0.5}^{+0.4}$ \\
\hline rxj1347 & $13.4_{-2.3}^{+2.3}$ & $23.4_{-2.1}^{+3.0}$ & $16.0_{-2.0}^{+2 . .3}$ & $13.8_{-0.8}^{+0.5}$ \\
\hline sersic159 & $9.7_{-0.7}^{+1.1}$ & $11.4_{-1.1}^{+1.1}$ & $5.2_{-0.6}^{+0.6}$ & $9.2_{-0.5}^{+0.9}$ \\
\hline zw3146 & $0.4_{-0.4}^{+2.3}$ & $25.9_{-2.2}^{+2.5}$ & $10.9_{-1.9}^{+1.9}$ & $15.9_{-0.9}^{+1.3}$ \\
\hline
\end{tabular}

Notes. ${ }^{(a)}$ Abbreviated cluster name. ${ }^{(b)}$ Measured average fractional spread of thermodynamic properties for the whole cluster (see Sect. 3.1, $M=1$ ) in per cent. 


\section{Appendix D: 2D Maps}
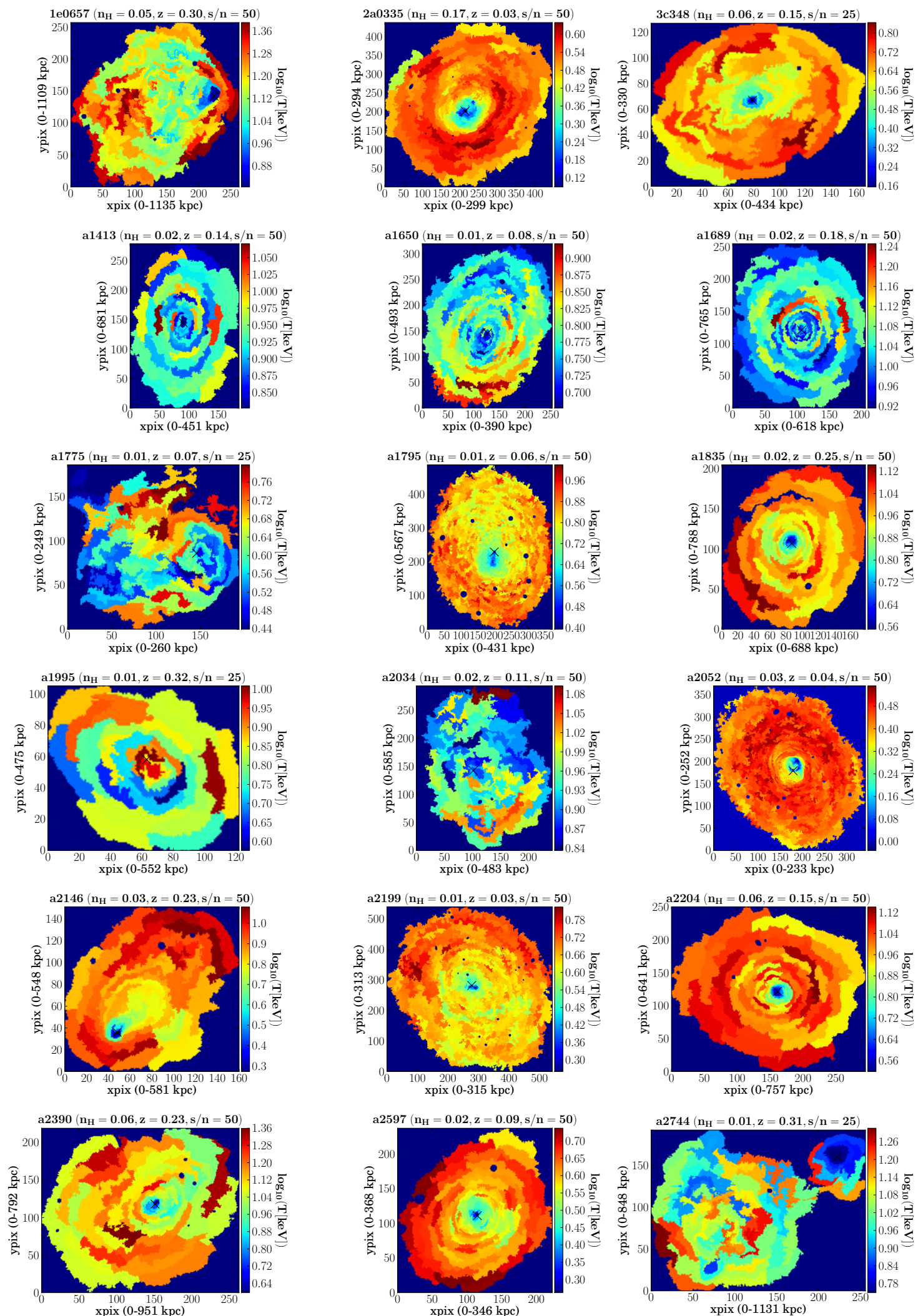

Fig. D.1. Temperature maps of all clusters in the sample. Bins have a $\mathrm{S} / \mathrm{N}$ of 50 or 25 (see Sect. 3.1) and temperatures are shown on a logarithmic colour scale. Excluding areas below the surface brightness cut (area-normalised normalisation $>10^{-7} \mathrm{~cm}^{-5} \operatorname{arcsec}^{-2}$ ) and where the errors on temperature are more than twice the best-fit temperature value. The plot title gives the abbreviated cluster name, the average foreground column density $n_{\mathrm{H}}\left[\mathrm{cm}^{-2}\right]$, and the redshift $z$. Scale: 1 pix $\sim 1^{\prime \prime}$. Crosses denote the peak of X-ray emission. 
F. Hofmann et al.: Thermodynamic perturbations in galaxy clusters
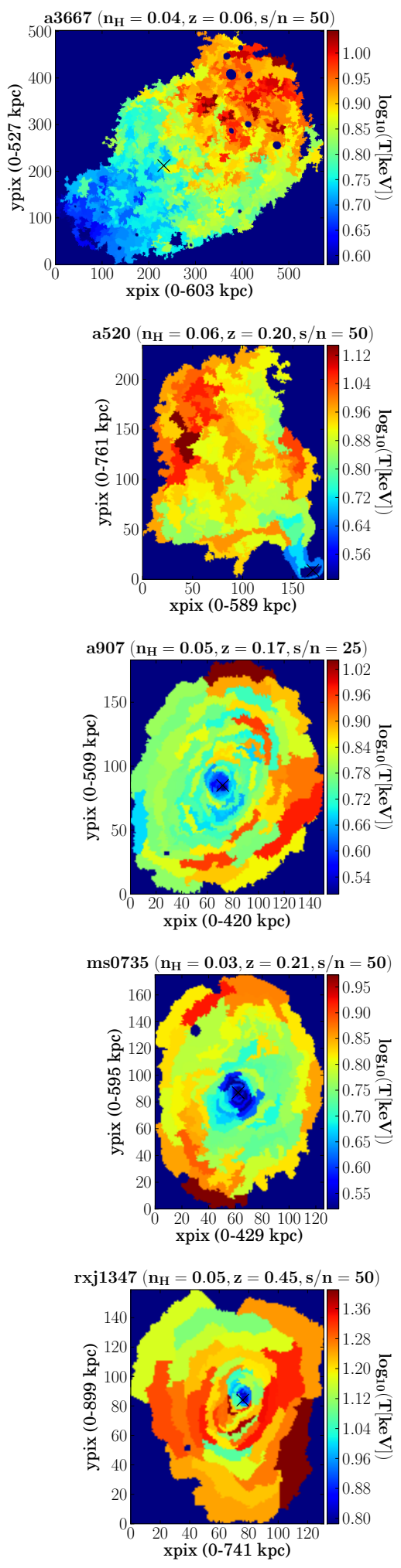

Fig. D.1. continued.
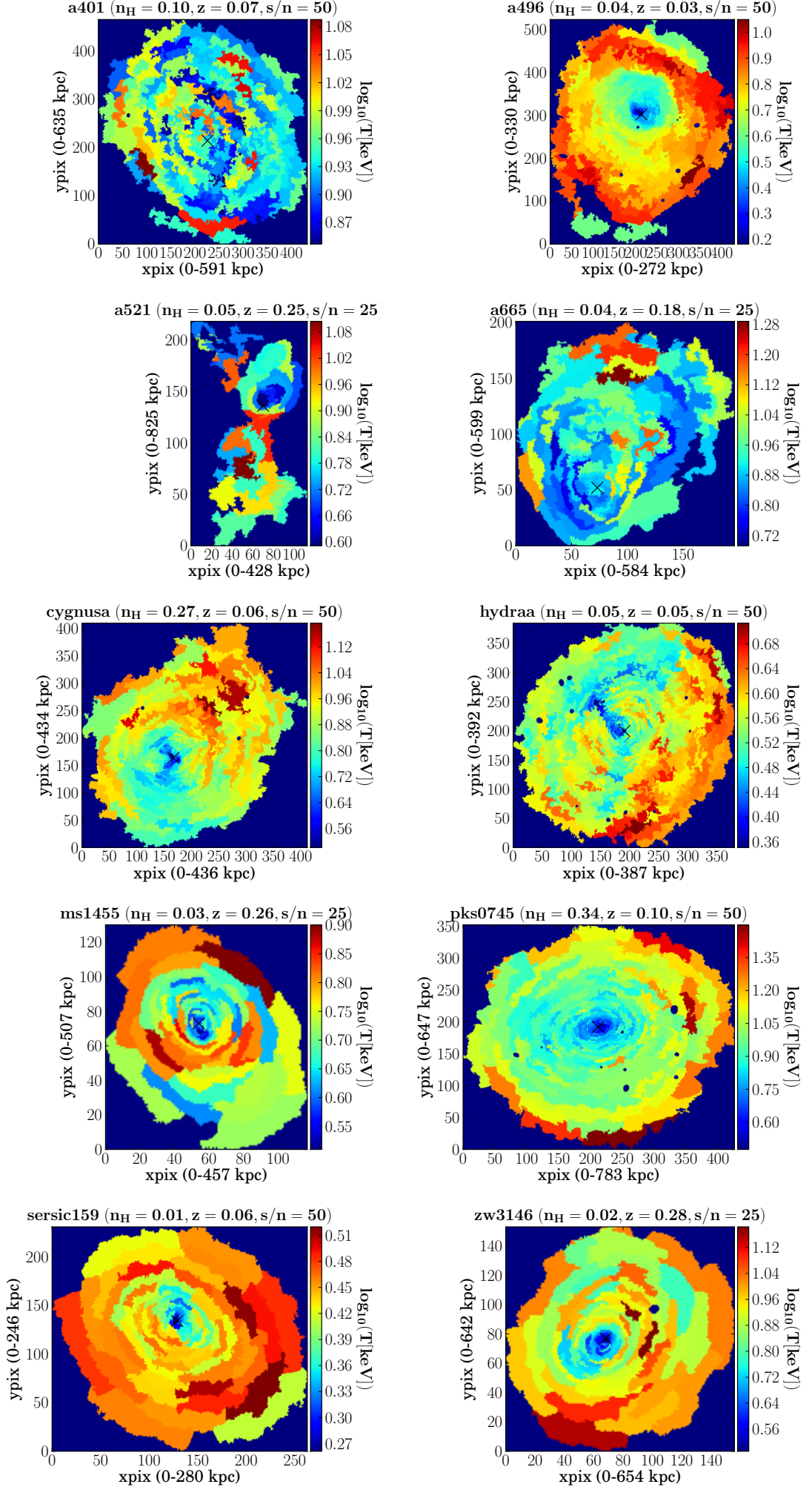
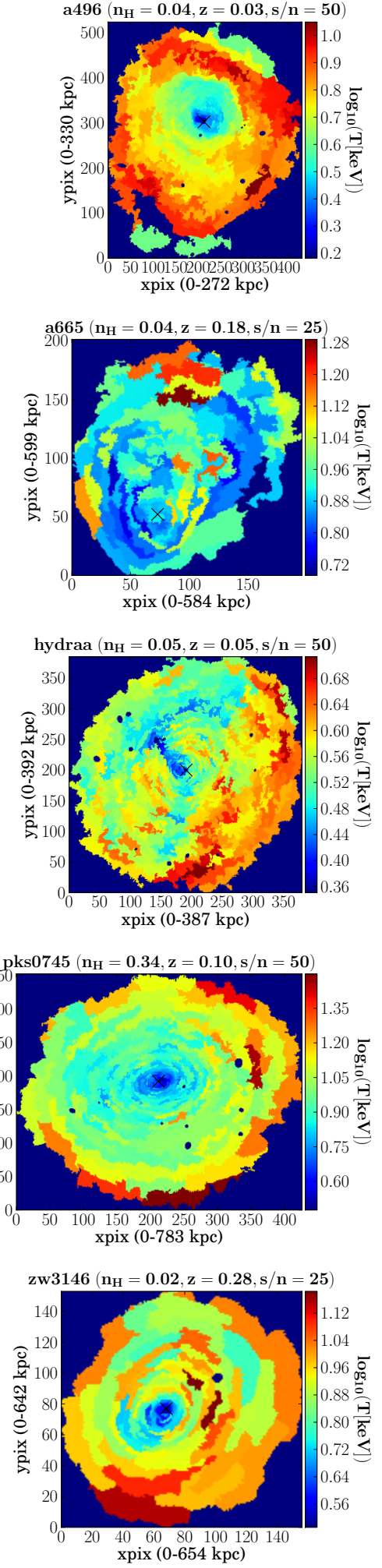


\section{Appendix E: Unsharp-masked count images}
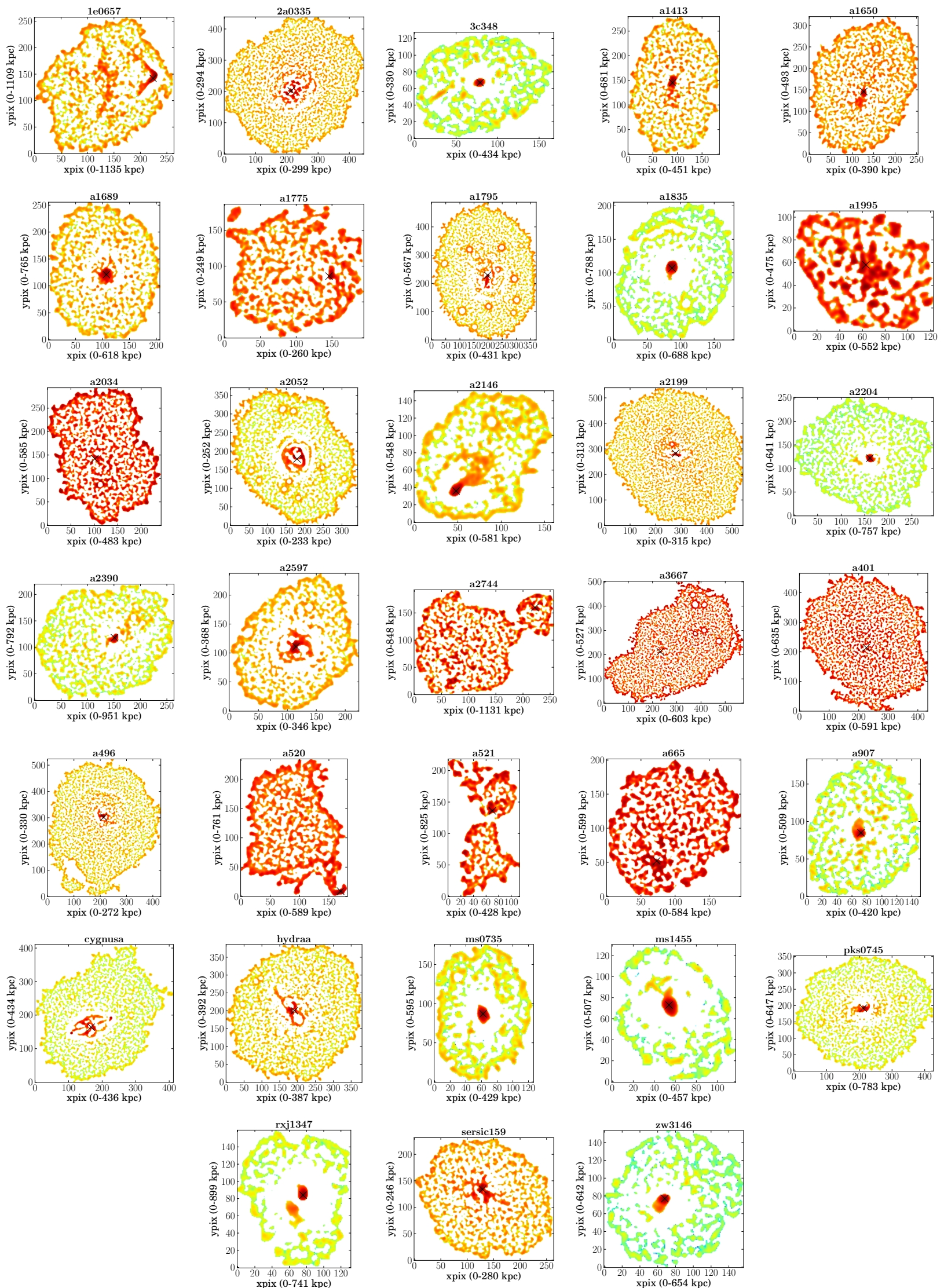

Fig. E.1. Unsharp-masked exposure-corrected images (logarithmic colour scale) of all clusters in the sample showing the difference between two count images smoothed with a Gaussian function (2 pixels and 5 pixels sigma). Colours indicate relative surface brightness differences (overdensities dark red, under-dense areas green to white). Scale: 1 pix $\sim 1^{\prime \prime}$. Crosses mark the peak of the X-ray emission. 


\section{Appendix F: Projected radial profiles}
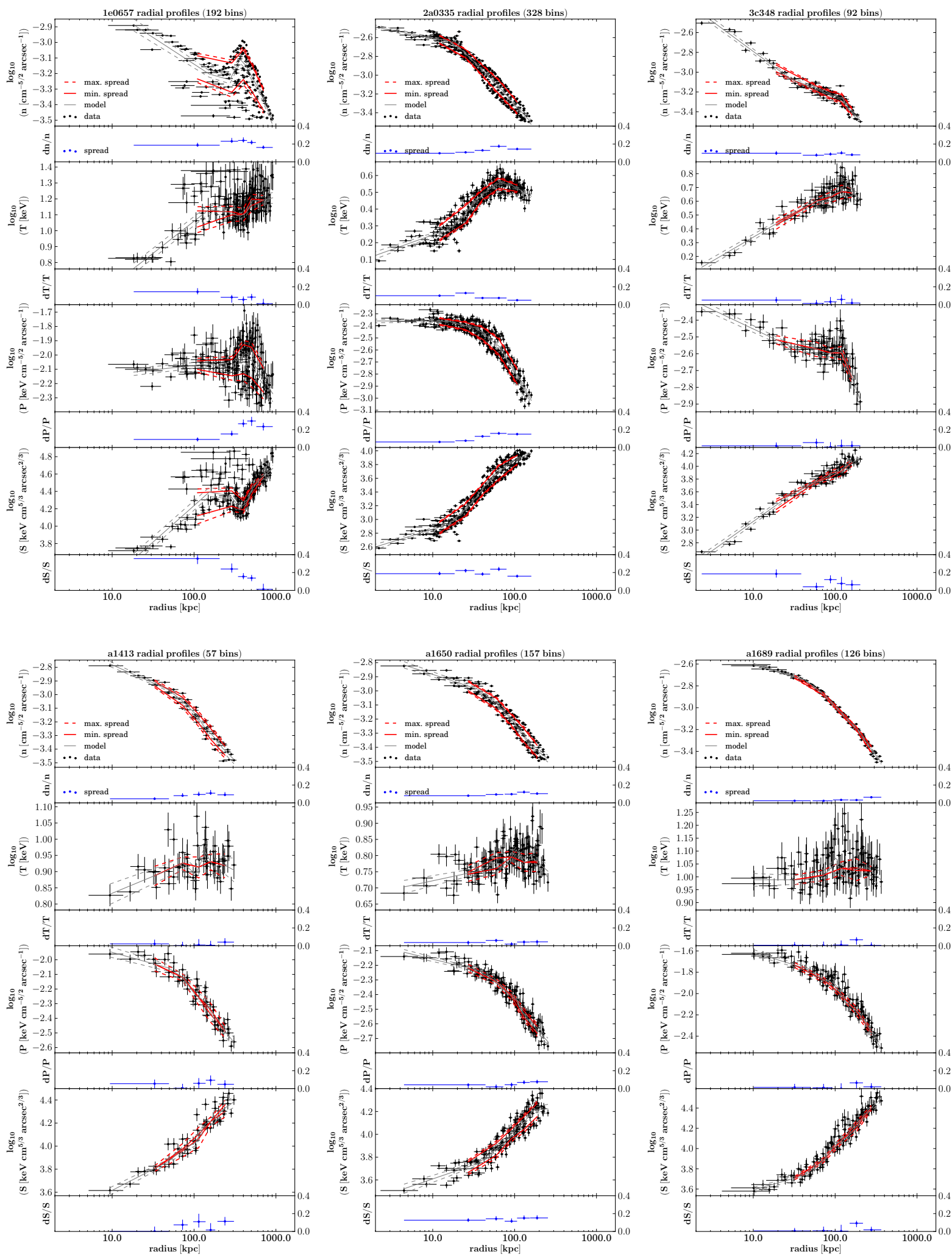

Fig. F.1. Radial profiles of projected density, temperature, pressure, and entropy. Cluster names are given in the plot titles. The cluster centres are marked as crosses in Figs. D.1 and E.1. Error bars are the fit errors and the standard deviation of the radial distribution for all spatial-spectral bins. The plotted lines show limits on intrinsic scatter around an average seven-node model (grey lines) within the given radial range (see Sect. 3.1). The small panels show the measured fractional scatter $(M=5)$ with confidence and radial range. 
A\&A 585, A130 (2016)
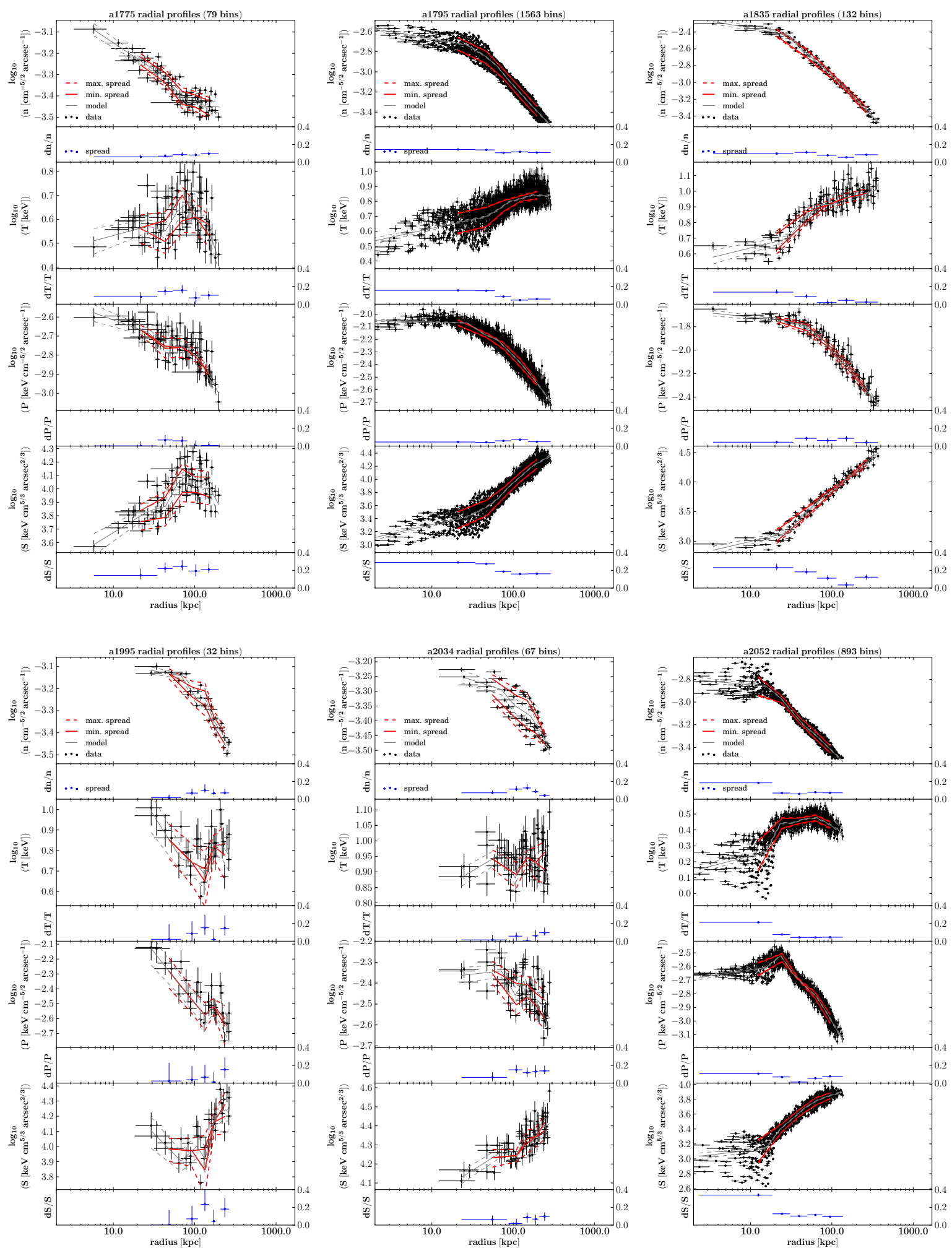

Fig. F.1. continued. 
F. Hofmann et al.: Thermodynamic perturbations in galaxy clusters
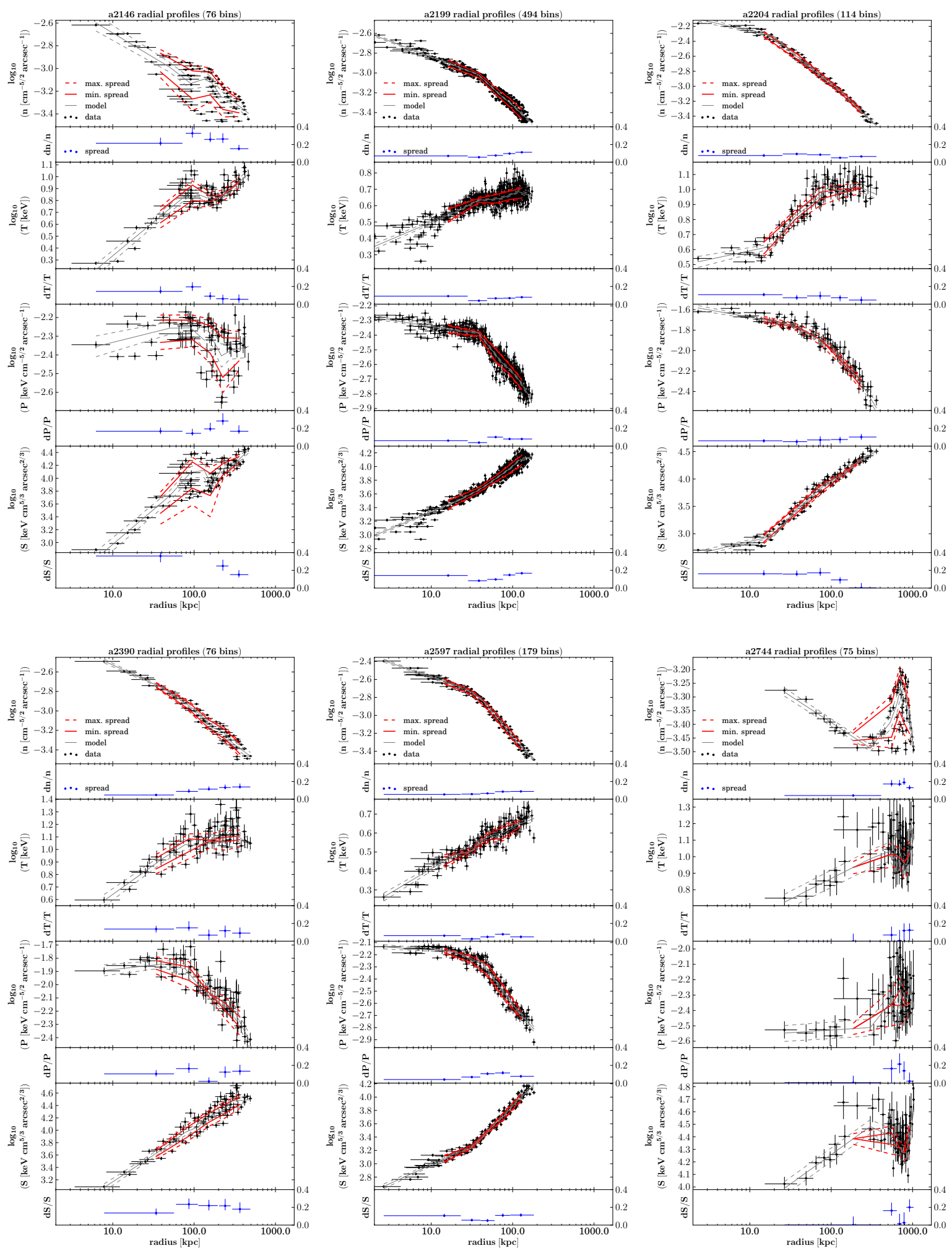

Fig. F.1. continued. 
A\&A 585, A130 (2016)
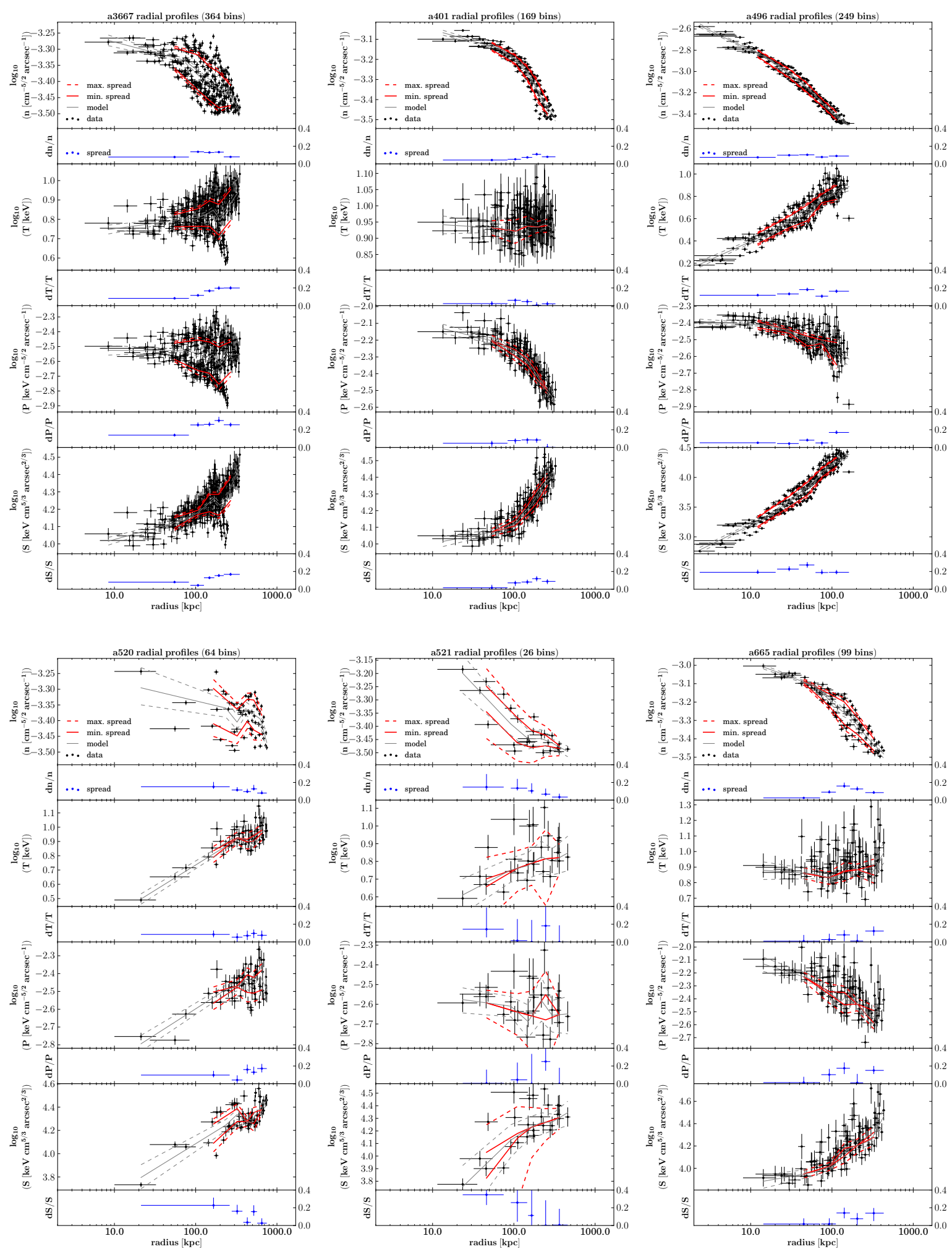

Fig. F.1. continued. 
F. Hofmann et al.: Thermodynamic perturbations in galaxy clusters
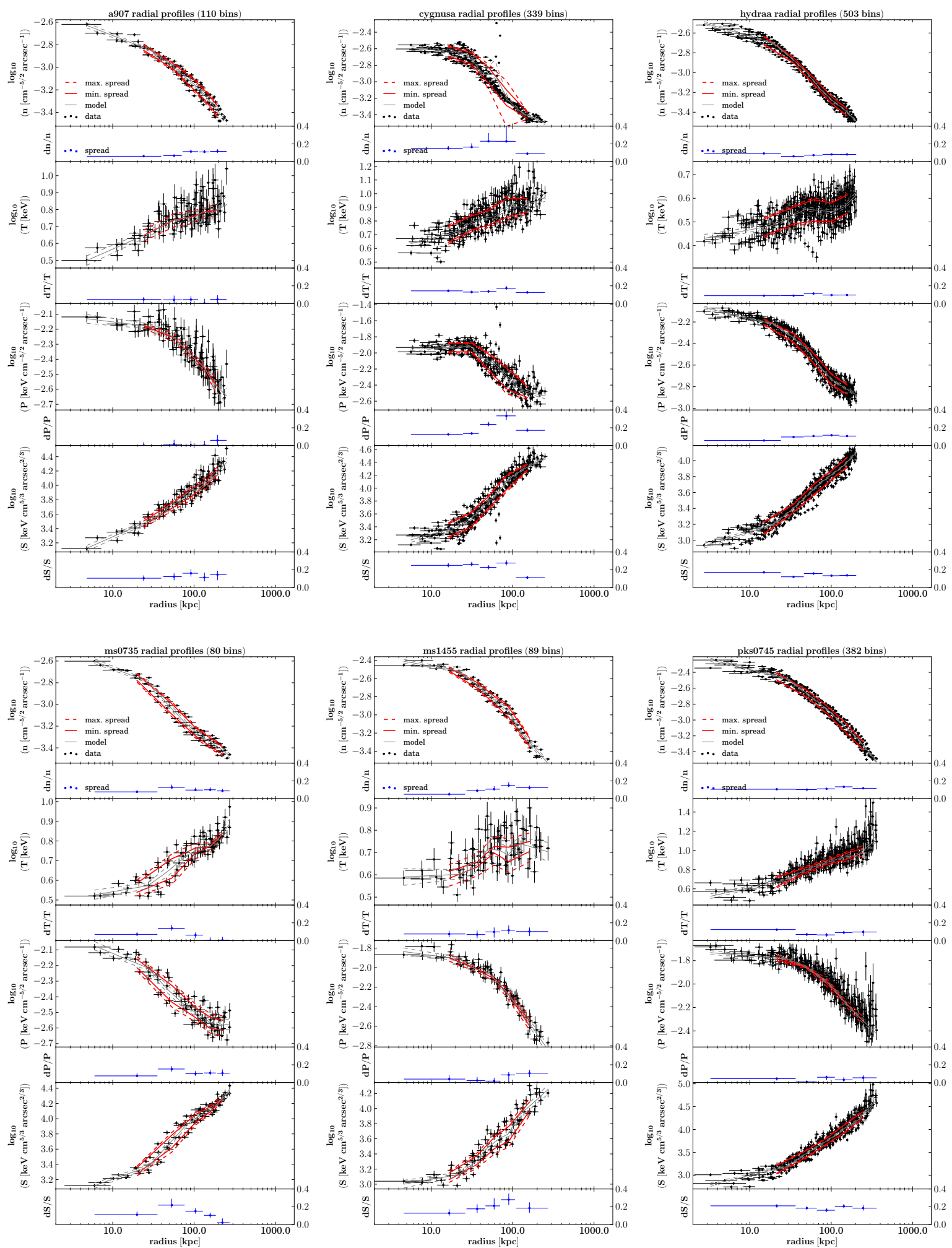

Fig. F.1. continued. 
A\&A 585, A130 (2016)
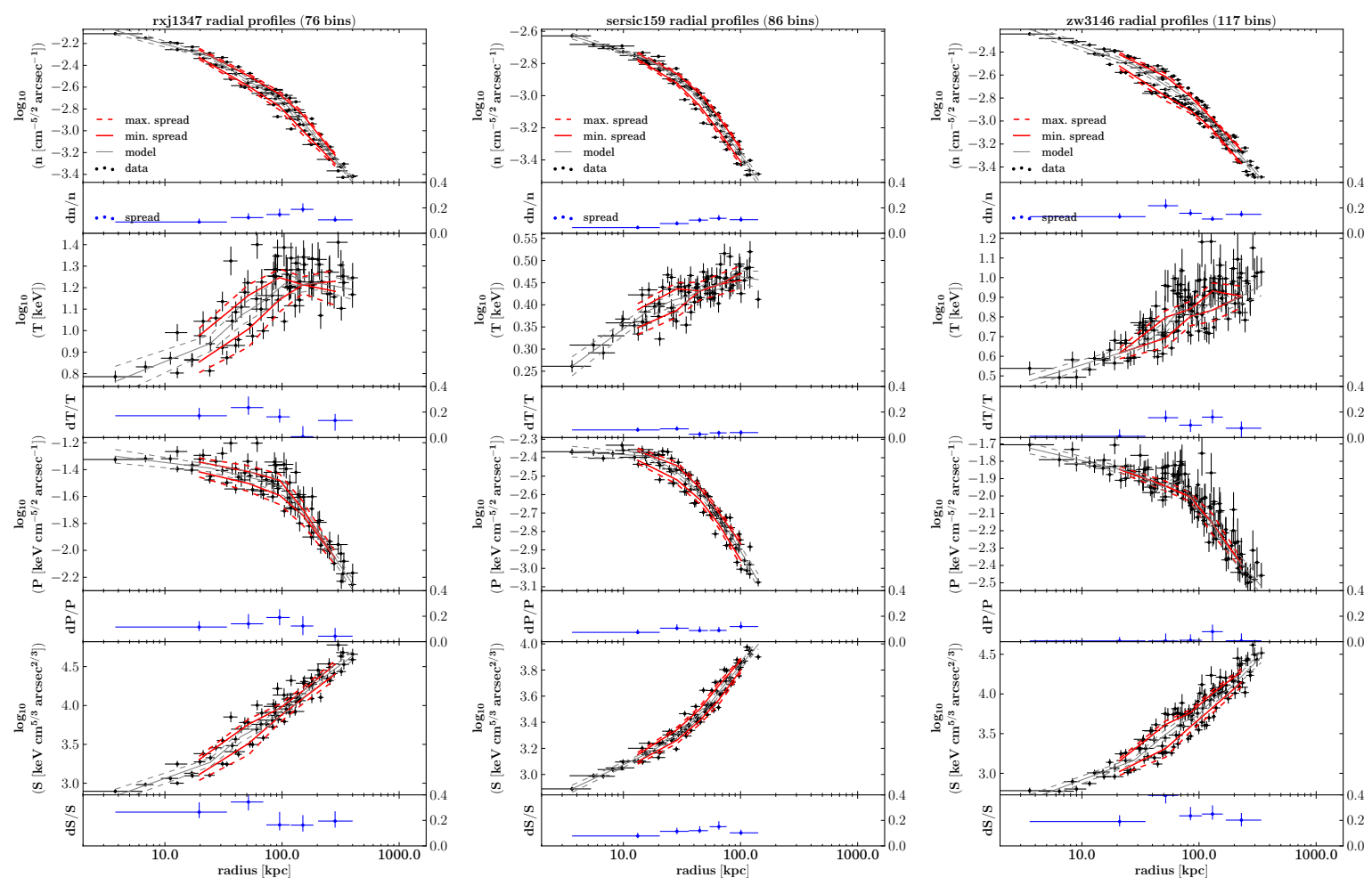

Fig. F.1. continued. 